\title{
Integration of Congestion and Awareness Control in Vehicular Networks
}

\author{
Miguel Sepulcre ${ }^{1}$, Javier Gozalvez ${ }^{2}$, Onur Altintas ${ }^{1}$ and Haris Kremo ${ }^{1}$ \\ ${ }^{1}$ TOYOTA InfoTechnology Center, Tokyo, Japan \\ ${ }^{2}$ UWICORE, Ubiquitous Wireless Communications Research Laboratory, Miguel Hernandez University of Elche \\ Elche (Alicante), Spain \\ msepulcre@jp.toyota-itc.com, j.gozalvez@umh.es, onur@jp.toyota-itc.com and hkremo@jp.toyota-itc.com
}

\begin{abstract}
Cooperative vehicular networks require the exchange of positioning and basic status information between neighboring nodes to support vehicular applications. The exchange of information is based on the periodic transmission/reception of 1-hop broadcast messages on the so called control channel. The dynamic adaptation of the transmission parameters when broadcasting such messages will be key for the reliable and efficient operation of vehicular networks. To this aim, vehicular networks utilize congestion control protocols to control the channel load, typically through the adaptation of the transmission parameters based on certain channel load metrics. Awareness control protocols are also required to adequately support cooperative vehicular applications. These protocols typically adapt the transmission parameters of periodic broadcast messages to ensure each vehicle's capacity to detect, and possibly communicate, with the relevant vehicles and infrastructure nodes present in its local neighborhood. To date, congestion and awareness control protocols have been normally designed and evaluated separately, although both will be required for the reliable and efficient operation of vehicular networks. In this context, this paper proposes and evaluates INTERN, a new control protocol that integrates two congestion and awareness control processes. The simulation results obtained for three different scenarios demonstrate that INTERN is able to satisfy the applications' requirements of all vehicles, while effectively controlling the channel load. The results obtained highlight the challenges ahead with emerging automated vehicles.
\end{abstract}

Keywords - Vehicular networks, congestion control, awareness control, cooperative ITS, connected vehicle.

\section{INTRODUCTION}

Cooperative vehicular networks are being designed to improve traffic safety and efficiency thanks to the real time exchange of information between vehicles (V2V - Vehicle-to-Vehicle) and between vehicles and infrastructure units (V2I - Vehicle-to-Infrastructure). The exchange of information is based on the periodic transmission/reception of 1-hop broadcast packets on the so called control channel using the IEEE $802.11 \mathrm{p}$ radio access technology in the $5.9 \mathrm{GHz}$ frequency band [1]. These packets are formally known as WSM (WAVE Short Messages) in the US or CAM (Cooperative Awareness Messages) in Europe, and are often referred to as beacons. Each packet includes positioning and basic status information of each vehicle, which is exploited by higher layer protocols and applications. For example, applications such as intersection collision warning or lane change assistance will exploit the position and speed information of nearby vehicles to detect potential road dangers with sufficient time for the driver to react. To effectively support vehicular safety applications, each vehicle needs to continuously receive updated information from all vehicles located within certain warning distance. The requirements of safety applications can be defined in terms of warning distance [2] and packet reception frequency (inverse of packet inter-reception time) [3]. Different applications can have different warning distance and packet reception frequency requirements [4], which can also depend on the context of the vehicles [5].

To adequately support cooperative vehicular applications, a number of awareness control protocols have been proposed in the literature [6]. These protocols are aimed at adapting the transmission parameters of beacons to ensure each vehicle's capacity to detect, and possibly communicate, with the relevant vehicles and infrastructure nodes present in its local neighborhood. In addition, all vehicles will periodically transmit their beacons on the control channel. This 
can lead to possible channel congestion, in particular under high traffic densities. The critical nature of the control channel has fostered significant efforts in the research and standardization communities to design congestion control protocols that ensure the scalability and adequate operation of vehicular networks by adapting the packet transmission frequency or power [6]. In fact, the ETSI communications architecture that future connected vehicles will implement includes a key Decentralized Congestion Control (DCC) module that is currently under development [7].

To date, congestion and awareness control protocols have been normally designed and evaluated separately, although both will be required for the reliable and efficient operation of vehicular networks. For example, in a highway scenario with a traffic jam in one direction of driving and free-flow conditions in the other direction, all vehicles might suffer channel congestion and would require the use of congestion control protocols to control the channel load. However, the requirements of the applications run by the vehicles in the traffic jam are notably lower from those of the applications run by the vehicles under free-flow conditions moving in the opposite direction with higher speeds and different inter-vehicle distances. In this scenario, a congestion control protocol would require the reduction of the transmission power and packet frequency to control the channel load, while an awareness control protocol would seek to increase the transmission parameters of vehicles under free-flow conditions due to their higher applications' requirements. In this context, an independent design (and disjoint operation) of congestion and awareness control techniques could create contradictory settings or conflicts that need to be solved [6]. To this aim, this paper proposes and evaluates INTERN (INTEgRatioN of congestion and awareness control), a new control protocol that integrates congestion and awareness control processes. INTERN dynamically adapts the transmission frequency and power of beacons of each vehicle to guarantee that its application's requirements are satisfied while controlling the channel load generated.

\section{STATE OF THE ART}

To effectively support vehicular safety applications, each vehicle needs to continuously receive updated information from its relevant neighboring vehicles. To this aim, different awareness control protocols that adapt the transmission parameters of beacons have been proposed in the literature [6]. For example, the work in [8] proposes OPRAM, an awareness control protocol that adapts each vehicle's transmission parameters to reliably and efficiently exchange a message before reaching a critical safety area such as an intersection. The work in [9] proposes an awareness control strategy based on the random adaptation of the transmission power and a control process that adapts the packet transmission frequency. The random adaptation of the power provides different reliability levels at different distances, while mitigating correlated packet collisions by randomizing them in space. The packet transmission frequency adaptation can reuse freed channel resources by further increasing the beacon frequency. The protocol proposed in [10] dynamically selects the power and data rate required to successfully transmit a packet to a given vehicle, based on estimations of the average signal attenuation using previously received beacons. In other studies such as [11], the packet transmission frequency is adapted to bind the tracking errors of surrounding vehicles. Considering multi-hop beaconing, the work in [12] proposes a fully distributed algorithm that decides whether a vehicle has to forward a received beacon or not. Such decision is taken by the vehicle itself and its objective is to maximize the reliability of the beacon transmission. However, other studies such as [13] demonstrated that single-hop beaconing is in general more efficient than multi-hop beaconing.

The periodic transmission of beacons occupies a significant portion of the control channel, which can easily get congested. Different congestion control protocols have been proposed in the literature to control the channel load by adapting the transmission parameters of beacons. Two of the most relevant congestion protocols available in the literature are LIMERIC [14] and PULSAR [15]. Both protocols propose the adaptation of the packet transmission frequency based on the experienced channel load, and set the transmission power to a fixed value. Both protocols are able to maintain the channel load below certain target threshold independently of the vehicular traffic density. LIMERIC and PULSAR are currently being discussed at ETSI's Technical Committee on ITS to be part of the DCC set of standards [16][17]. Other congestion control approaches are also available in the literature. For example, the proposal in [18] computes the transmission power of each vehicle based on the number of detected neighboring vehicles and other metrics such as the estimated carrier sensing range. The objective of the protocol proposed in [19] is the efficient transmission of beacons as frequently as possible, while maintaining a congestion-free wireless channel. To this aim, in [19] the packet transmission frequency is adapted by taking into account the channel quality (estimated based on observed packet collisions, Signal-to-Noise ratio and number of neighboring vehicles) and the message utility. The work in [20] recently proposed the adaptation of the packet transmission frequency as a function of the channel load experienced, the target channel load and the number of neighboring vehicles. Other protocols such as the one 
proposed in [21] consider the adaptation of the contention window size of IEEE 802.11p. In particular, the work in [21] proposes the adaptation of the minimum contention window size based on local transmission statistics that estimate the channel load and differentiating traffic priorities. Other congestion control protocols propose sensing the wireless channel and reducing the transmission power when a channel load threshold is exceeded [22], or when the number of messages in the MAC queue is above certain maximum level [23].

Congestion and awareness control protocols have been typically designed to achieve fairness. Local fairness is achieved when neighboring nodes present similar performance/configuration. Global fairness is achieved when the minimum performance/configuration of the network is maximized [15], i.e. when the performance/configuration of the vehicle under the most adverse conditions is maximized. Congestion control protocols have been designed to provide fairness in terms of channel load and transmission parameters. However, awareness control protocols should be able to provide fairness at the application level, i.e. fairness in terms of the capacity to satisfactorily meet the applications' requirements (i.e. the applications' effectiveness).

When congestion and awareness protocols operate independently, negative interactions or conflicts can arise, which can provoke unintended and negative adaptation loops that reduce the network and system performance. Limited efforts have been conducted to jointly consider congestion and awareness control processes. In this context, this paper proposes INTERN, a protocol that integrates different congestion and awareness control techniques. In particular, INTERN integrates control mechanisms inspired by LIMERIC and PULSAR congestion control protocols with the awareness control design policy proposed in [24]. The policy proposed in [24] considers that each vehicle proactively adapts its transmission parameters to the minimum needed to satisfy its application's requirements. This paper is an extension of our previous work [25].

\section{CONSIDERED CONGESTION AND AWARENESS CONTROL PROTOCOLS}

\section{A. Congestion control}

LIMERIC [14] adapts the packet transmission frequency of each vehicle based on a target channel load level and the channel load it locally measures every time window. The packet transmission frequency of vehicle $j$ at time instant $t$ is calculated with the following equation:

$$
r_{j}(t)=(1-\alpha) r_{j}(t-1)+\beta\left(r_{g}-r(t-1)\right)
$$

where $r_{g}$ is the overall target packet frequency, $r(t-1)$ represents the measured overall packet frequency by the vehicle in the previous time window, and $\alpha=0.1$ and $\beta=1 / 150$ are system constants. In practical implementations, vehicles can measure the load in terms of Channel Busy Ratio $(C B R)$, i.e. the fraction of time that the channel is sensed as busy. $r_{g}$ and $r(t-1)$ can then be replaced by $C B R_{\max }$ and $C B R(t-1)$, respectively, with $C B R_{\max }$ representing the target channel busy ratio and $C B R(t-1)$ the channel busy ratio measured by vehicle $j$. As noted in [14], $\alpha$ and $\beta$ have a high influence on the system's stability that is represented by the capacity to reach a solution in which the transmission parameters of vehicles are stable if the conditions remain constant (e.g. traffic density conditions). A stable system operation ensures that the convergence point, i.e. the channel busy ratio at which the system converge in a stable situation and the convergence speed, i.e. the velocity with which the final solution (channel busy ratio and transmission parameters of each vehicle) have been reached. LIMERIC has been shown to provide high accuracy and stability in scenarios where all the nodes measure the same channel load when utilizing an additional mechanism that establishes a maximum gain in equation (1) [14].

PULSAR [15] is a congestion control protocol that adapts the packet transmission frequency of each vehicle using an Additive Increase Multiplicative Decrease (AIMD) technique. PULSAR proposes that each vehicle piggybacks its locally experienced $C B R$ and the maximum $C B R$ experienced by its neighbors since the transmissions from each vehicle can influence the load up to a distance of approximately two hops. Each vehicle adapts its packet transmission frequency based on the maximum $C B R$ among its experienced $C B R$ and the $C B R$ levels reported by its neighbors (i.e. the maximum $C B R$ within two hops). If the resulting $C B R$ is above $C B R_{\max }$, the packet transmission frequency is decreased by a multiplicative factor; otherwise the packet transmission frequency is increased by an additive factor. Additionally, the multiplicative and additive factors are modified depending on whether the current packet transmission frequency of the vehicle is above or below the packet transmission frequency of neighboring vehicles. To this aim, each vehicle periodically calculates the average packet transmission frequency of the neighboring vehicles; each vehicle also 
piggybacks in its beacons its packet transmission frequency. This mechanism and the AIMD process ensure that vehicles within two hops distance converge to the same packet transmission frequency.

A congestion control protocol that combines LIMERIC and PULSAR is being discussed within the ETSI standardization process [16][17]. The combined scheme uses LIMERIC's linear control process and PULSAR's CBR information exchange. The combined protocol (referred to as LIMERIC+PULSAR) is used as benchmark in this study.

\section{B. Awareness control}

This study implements the MINT (minimum packet transmission frequency) awareness control protocol that follows the design policy proposed in [24]. Following this policy, each vehicle proactively adapts its transmission parameters to the minimum needed to satisfy its individual application's requirements. An application is satisfactorily supported if the number of beacons correctly received per second at the established warning distance is higher than the application's requirement in terms of packet reception frequency. MINT sets the packet transmission frequency equal to the application's packet reception frequency plus a fixed margin $\Delta T_{f}=1 \mathrm{~Hz}$. The transmission power is then set to the level needed to ensure that the demanded packet reception frequency is guaranteed at the application's warning distance. To this aim, MINT identifies the transmission power needed utilizing the analytical model that relates the Packet Delivery Ratio (PDR) with the transmission power and the distance between transmitter and receiver proposed in [26].

\section{INTERN - INTEGRATION OF CONGESTION AND AWARENESS CONTROL}

INTERN aims to configure the transmission parameters of each vehicle so that its applications' requirements can be satisfied and the channel load can be maintained below the target $C B R$. In this context, all vehicles implementing INTERN will tend to use the minimum transmission settings that satisfy their individual application's requirements under high traffic density conditions. On the other hand, INTERN will enable vehicles to increase their transmission settings under low traffic densities so that the target $C B R$ is reached and the channel is fully utilized. To achieve its objectives, INTERN integrates MINT with a control process inspired by LIMERIC and PULSAR. INTERN exploits the benefits of the 2-hops piggybacking proposed in PULSAR in order to achieve global fairness. Once INTERN configures the transmission frequency, it calculates the transmission power following the MINT protocol: the transmission power is set to the minimum power level needed to ensure that the demanded packet reception frequency is guaranteed at the application's warning distance.

INTERN configures the packet transmission frequency of each vehicle, $T_{f}$, as the minimum required by its application, $R$, plus certain margin $\Delta T_{f}$.

$$
T_{f}=R+\Delta T_{f}
$$

The $\Delta T_{f}$ parameter is dynamically calculated by each vehicle using a control process. LIMERIC proposes to adapt $T_{f}$ so that nearby vehicles are assigned similar packet transmission frequency values (and hence are allocated similar bandwidth). Instead, INTERN adapts $\Delta T_{f}$ to ensure that the individual application's requirements of each vehicle are satisfied. The control process is designed so that the channel load is maintained below the target $C B R$ level $C B R_{\max } . \Delta T_{f}$ is then adapted as a follows:

$$
\Delta T_{f}=\Delta T_{f}^{T}+\frac{\Delta T_{f}^{T}}{C B R_{2 h o p s}}\left(C B R_{\max }-C B R_{2 h o p s}\right)=\Delta T_{f}^{T} \frac{C B R_{\max }}{C B R_{2 h o p s}}
$$

$C B R_{2 h o p s}$ is the maximum $C B R$ experienced within two hops and is obtained following the PULSAR approach. To this aim, INTERN requires that each vehicle attaches to each beacon its locally experienced $C B R$ and the maximum $C B R$ experienced by its neighboring vehicles. Vehicles implementing INTERN increase $\Delta T_{f}$ (following eq. (3)) when $C B R_{\max }$ $>C B R_{2 h o p s}$. Similarly, vehicles decrease $\Delta T_{f}$ when $C B R_{\max }<C B R_{2 h o p s}$.

To achieve fairness at the application level, all vehicles should have a similar $\Delta T_{f}$, i.e. they should apply similar increments of the packet transmission frequency with respect to the minimum they need. To ensure this is actually the case for vehicles within two hops distance, INTERN applies the PULSAR piggybacking scheme to the $\Delta T_{f}$ parameter. As a result, each vehicle attaches to its beacons its current $\Delta T_{f}$ and the minimum $\Delta T_{f}$ received from its neighboring vehicles. $\Delta T_{f}^{T}$ in equation (3) is then calculated by each vehicle as the minimum $\Delta T_{f}$ reported by its neighbors. Thanks to this mechanism, vehicles will rapidly adapt to variable traffic density conditions. 
We consider minimum and maximum values of $1 \mathrm{~Hz}$ and $3 \mathrm{~Hz}$ (different minimum and maximum values could be considered) for the $\Delta T_{f}$ parameter so that the application's requirements can be satisfied but we avoid operating with packet transmission frequencies much higher than the ones required by the applications. The minimum packet transmission frequency of a vehicle will be therefore at least $1 \mathrm{~Hz}$ above the minimum required by its application.

\section{PERFORMANCE EVALUATION}

The performance of INTERN has been evaluated using Matlab in different scenarios and considering different traffic densities. The results obtained have been compared to the ones obtained with MINT and the congestion control protocol that combines LIMERIC and PULSAR (LIMERIC+PULSAR).

\section{A. Evaluation scenarios}

Three evaluation scenarios have been selected to demonstrate the benefits of INTERN:

- Scenario 1 - intersection with LOS (Fig. 1a). In this scenario, vehicles are uniformly distributed on the roads shown in Fig. 1a. The scenario is characterized by the traffic density in vehicles $/ \mathrm{km} / \mathrm{lane}$, and the length of each one of the four roads $(3.5 \mathrm{~km})$. This scenario does not consider buildings at intersection corners, and therefore all vehicles have LOS (Line-of-Sight) propagation conditions.

- Scenario 2 - intersection with NLOS (Fig. 1b). This scenario is similar to scenario 1, but considers the presence of buildings blocking the radio signal at intersection corners. The obstructing buildings have an important effect on the experienced propagation losses and the quality of vehicular communications [27]. In this scenario, vehicles that are close to the intersection can detect and receive messages from vehicles in all four intersecting streets. The obstruction caused by buildings results in that the rest of vehicles can only detect vehicles on the same street, and therefore experience a significantly lower channel load.

- Scenario 3 - highway (Fig. 1c). This scenario is aimed at evaluating the impact of the movement and speed of vehicles. In particular, this third scenario is used to evaluate the convergence and stability properties of the protocols when two groups of vehicles approach each other as illustrated in Fig. 1c. Each group of vehicles is $2 \mathrm{~km}$ long, occupies 2 lanes and is characterized by a uniform traffic density. All vehicles move at a constant speed of $120 \mathrm{~km} / \mathrm{h}$. The length of the road is $7 \mathrm{~km}$, and the two groups of vehicles get perfectly aligned next to each other after $t=75$ seconds.

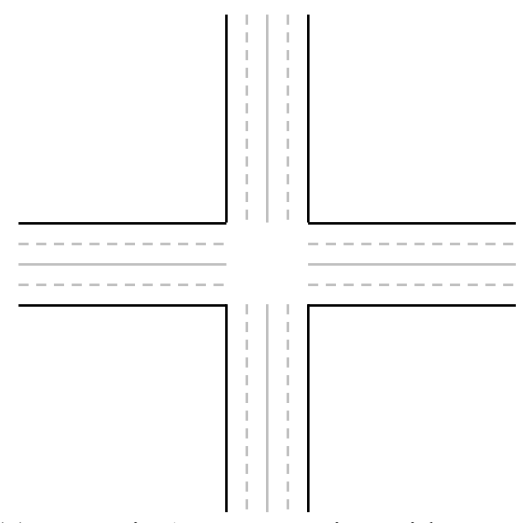

(a) Scenario 1 - Intersection with LOS

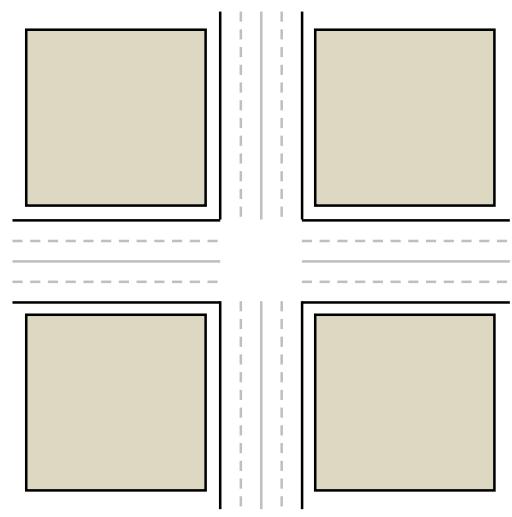

(b) Scenario 2 - Intersection with NLOS

Fig. 1. Evaluation scenarios.

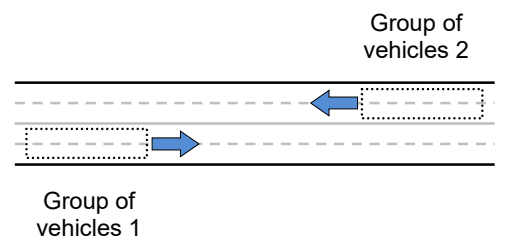

(c) Scenario 3 - Highway

All vehicles in the three scenarios periodically broadcast beacons and dynamically adapt their transmission parameters following the operation of the protocol under evaluation. All vehicles start using the same transmission power (by default $P_{t}=33 \mathrm{dBm}$ ) and a random packet transmission frequency $\left(T_{f}\right)$ between $1 \mathrm{~Hz}$ and $10 \mathrm{~Hz}$. Following the indications provided in [26], the Nakagami- $m$ propagation model has been employed with $m=3$. 
Each vehicle in all three scenarios runs a vehicular application that requires that at least $R$ beacons are correctly received per second by all vehicles within a given warning distance, $W_{d}$. To avoid limiting this study to a particular application, different combinations of application warning distance and required packet reception frequency have been considered. A first analysis has been performed considering that each vehicle sets its initial application requirements randomly, with the warning distance varying between 50 and $200 \mathrm{~m}$ and the packet reception frequency varying between $1 \mathrm{~Hz}$ and $10 \mathrm{~Hz}$. From these initial settings, the application requirements linearly vary during the simulation. A second analysis has been conducted considering constant and equal application requirements for all vehicles.

The capacity of each protocol to satisfy the application requirements is evaluated using the $D_{p}$ metric. This metric represents the difference between the packet reception frequency demanded by the application and the number of packets that are actually received per second at the applications' warning distance. The requirements of an application are therefore satisfied if $D_{p}>0$. The $D_{p}$ values reported in this paper have been calculated taking into account packet losses due to propagation conditions.

$C B R_{\max }$ has been set to 0.6 following [14] that indicates that this is the $C B R$ value that maximizes the throughput or number of successful messages exchanged per second. The $C B R$ values computed in this study have been obtained from the aggregation of all packets sensed by each vehicle's radio interface, i.e. with a received signal higher than $90 \mathrm{dBm}$. Table I summarizes some of the most relevant communications and simulation parameters.

TABLE I. COMMUNICATIONS AND SIMULATION PARAMETERS

\begin{tabular}{|l|l|}
\hline Parameter & Value \\
\hline Packet size $[$ Bytes] & 250 \\
\hline Min. and Max. transmission power $[\mathrm{dBm}]$ & -10 and 33 \\
\hline Min. and Max. packet transmission frequency $[\mathrm{Hz}]$ & 1 and 20 \\
\hline Min. and Max. $\Delta T_{f}[\mathrm{~Hz}]$ & 1 and 3 \\
\hline Data rate $[\mathrm{Mbps}]$ & 6 \\
\hline Carrier sense threshold $[\mathrm{dBm}]$ & -90 \\
\hline Reception threshold $[\mathrm{dBm}]$ & -82 \\
\hline Target channel busy ratio $\left(C B R_{\max }\right)$ & 0.6 \\
\hline$C B R$ measurement period $[\mathrm{ms}]$ & 250 \\
\hline Warning distance required $\left(W_{d}\right)[\mathrm{m}]$ & $50-200$ \\
\hline Packet reception frequency required $(R)[\mathrm{Hz}]$ & $1-10$ \\
\hline Traffic density $[$ vehicles/km/lane] & $50,75,100$ \\
\hline Simulation time $[\mathrm{s}]$ and simulation runs & 150 and 10 \\
\hline
\end{tabular}

\section{B. Scenario 1 - intersection with LOS}

Scenario 1 has been mainly utilized to evaluate the spatial distribution of the channel load and the application's effectiveness $\left(D_{p}\right)$. Figures 2 and 3 plot the average spatial distribution of the $C B R$ and $D_{p}$, respectively, as a function of the distance of the vehicles to the intersection; the results are reported for different traffic densities. The vertical lines in Figures 2 and 3 represent the $5^{\text {th }}$ and $95^{\text {th }}$ percentiles.

The MINT results in Fig. 2 show that high $C B R$ levels are obtained at short distances to the intersection since the number of neighboring vehicles increases as a vehicle approaches the intersection. Additionally, the $C B R$ experienced with MINT increases with the traffic density. This is the case because MINT configures each vehicle with the minimum transmission power and frequency required to satisfy its application requirements, and the protocol does not control the channel load. This results in that the application requirements are satisfied with a constant $D_{p}$ metric independently of the traffic density (see Fig. 3). Under low and medium traffic densities, the use of the minimum required transmission settings also results in that the channel is not utilized to its full capacity since the maximum experienced $C B R$ is below 
$C B R_{\max }$. The experienced $C B R$ with MINT only surpasses $C B R_{\max }$ under the highest simulated traffic density and for vehicles close to the intersection. This result demonstrates that in this situation it is not possible to satisfy the application requirements of all vehicles without exceeding $C B R_{\max }$.

Fig. 2 shows that LIMERIC+PULSAR is able to strictly maintain the $C B R$ experienced below $C B R_{\max }$ irrespective of the traffic density and the transmission power; Fig. 2 shows the results achieved with 3 fixed transmission power levels. Vehicles use a constant transmission power but dynamically adapt their packet transmission frequency to the channel load. When vehicles utilize the maximum transmission power $\left(P_{t}=33 \mathrm{dBm}\right.$ in this study), they are able to detect other vehicles at large distances, and therefore the number of detected vehicles increases. In this context, LIMERIC+PULSAR results in a packet transmission frequency of around $2 \mathrm{~Hz}$ when $P_{t}$ is equal to $33 \mathrm{dBm}$. This results in negative $D_{p}$ values for nearly all vehicles when utilizing a transmission power of $33 \mathrm{dBm}$ (see Fig. 3). Reducing the transmission power decreases the vehicles' transmission range and enables the use of higher packet transmission frequencies with LIMERIC+PULSAR. This results in that LIMERIC+PULSAR experiences higher $D_{p}$ values as the transmission power is reduced (Fig. 3). For $P_{t}=23 \mathrm{dBm}$, vehicles located at longer distances to the intersection can achieve positive $D_{p}$ values since they are able to use higher packet transmission frequencies compared to vehicles close to the intersection. For $P_{t}=13 \mathrm{dBm}$, Fig. 3 shows that a high percentage of vehicles experience positive $D_{p}$ values, in particular under low traffic densities. LIMERIC+PULSAR provides similar packet transmission frequencies to nearby vehicles irrespective of their application requirements. These results emphasize the need for the integration of congestion and awareness control protocols so that the requirements of the maximum possible number of vehicles are satisfied while efficiently utilizing the channel capacity.

Fig. 2 also illustrates the average spatial distribution of the $C B R$ experienced when implementing the new proposal INTERN. Rather than configuring vehicles with the minimum transmission settings required, INTERN increases them until the $C B R_{\max }$ level is reached at the center of the intersection. Fig. 2 shows that INTERN can maintain the $C B R$ below $C B R_{\max }$ in the scenarios with low and medium traffic densities. INTERN does not increase the transmission settings of vehicles experiencing low $C B R$ values so that vehicles close to the intersection do not experience a $C B R$ level above $C B R_{\max }$. This is possible thanks to the piggybacking process incorporated into INTERN. When the traffic density increases to $100 \mathrm{veh} / \mathrm{km} / \mathrm{lane}$, the vehicles close to the intersection use the minimum transmission power and packet frequency required to satisfy their application requirements. This results in that these vehicles experience with INTERN the same $C B R$ (Fig. 2) and $D_{p}$ values (Fig. 3) they would experience with MINT. This feature demonstrates the capacity of INTERN to adapt its operation to the traffic density conditions and experienced load levels. The results in Fig. 3 also show that when the traffic density decreases, vehicles can increase their packet transmission frequency to fully utilize the channel; this results in higher $D_{p}$ levels. Higher $D_{p}$ levels reduce the risks provoked by sudden or unexpected channel variations that could result in that the transmission settings are not capable of guaranteeing the application requirements. 
This is an author-created postprint version. The final publication is available at: https://www.sciencedirect.com/science/article/pii/S1570870515002279
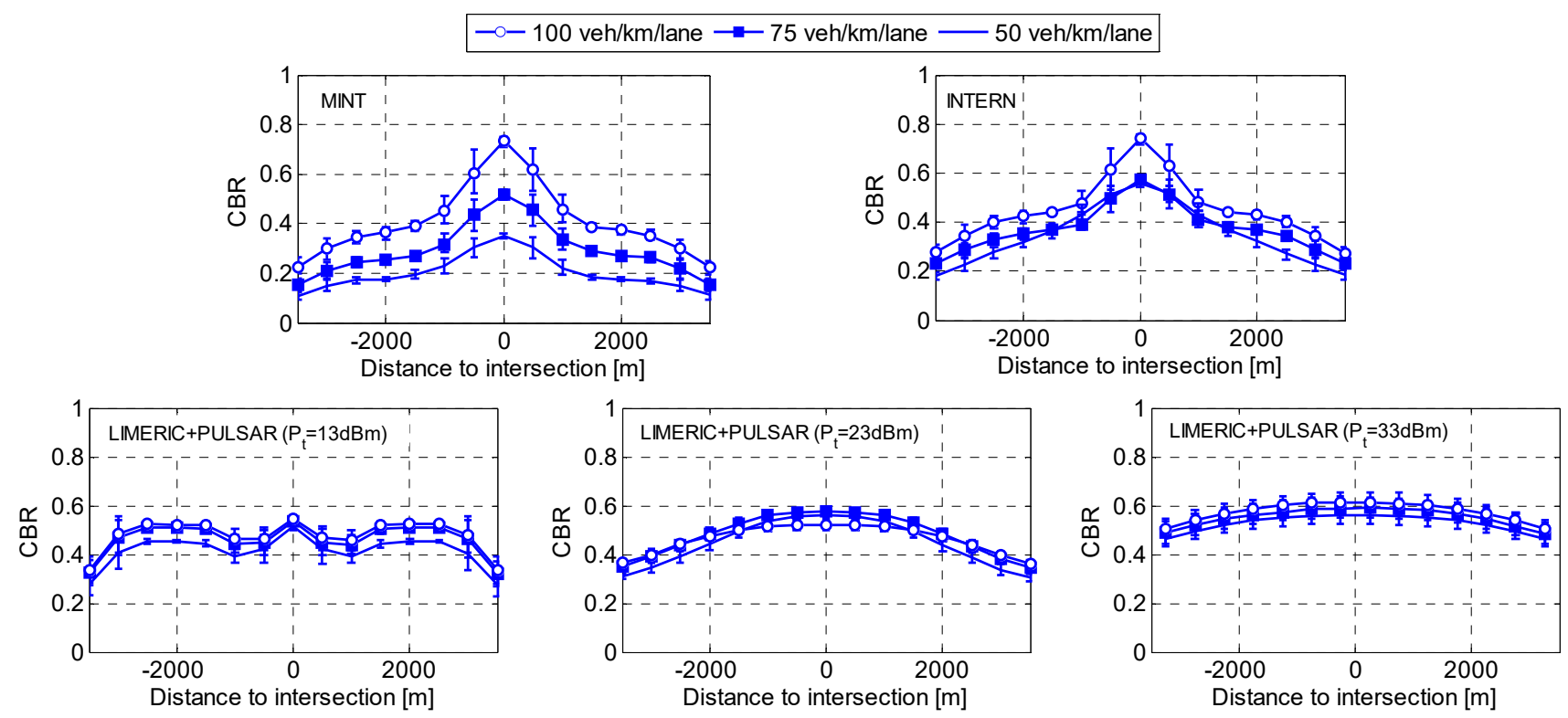

Fig. 2. Average spatial distribution of the $C B R$ (Channel Busy Ratio) under different traffic densities in scenario 1 (intersection with LOS). The vertical lines represent the $5^{\text {th }}$ and $95^{\text {th }}$ percentiles.
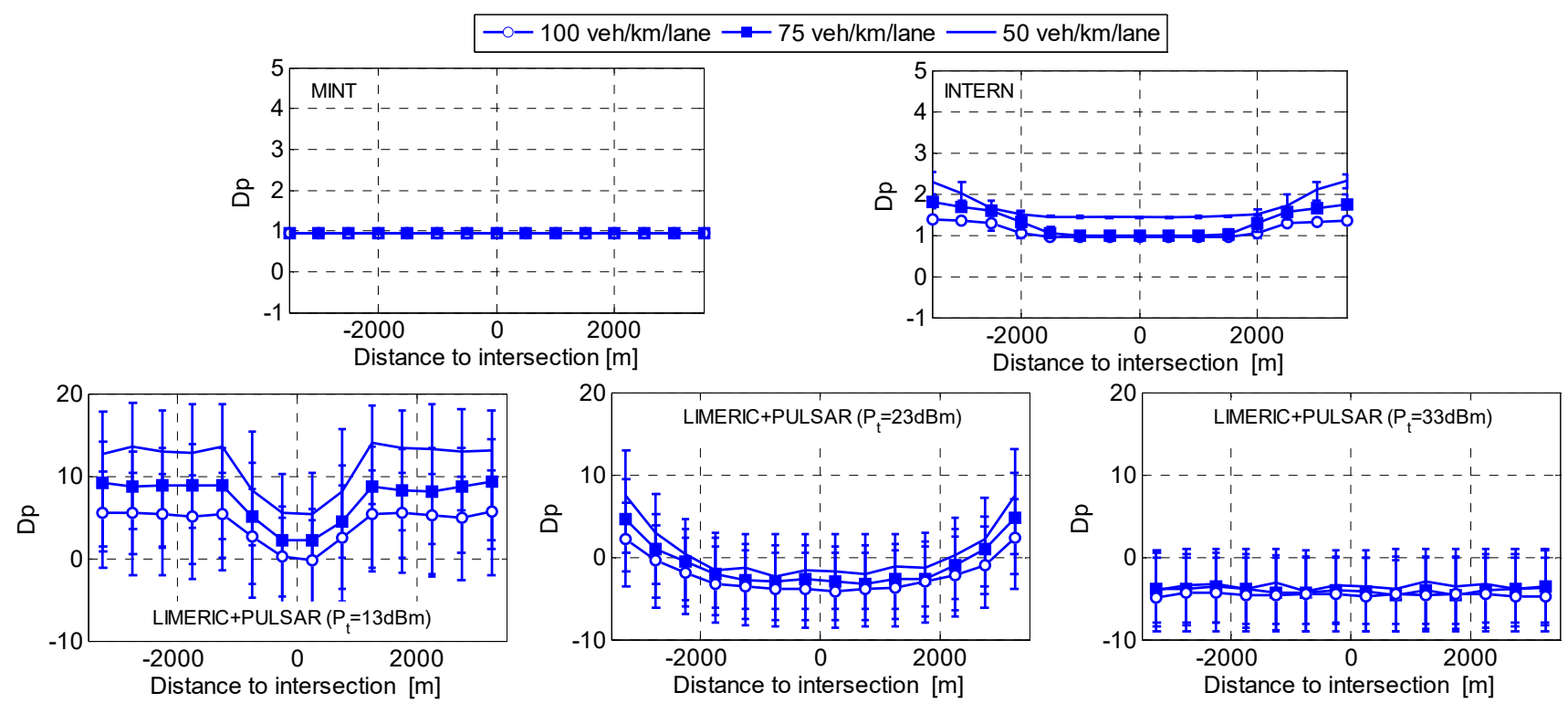

Fig. 3. Average spatial distribution of the $\mathrm{D}_{\mathrm{p}}$ metric under different traffic densities in scenario 1 (intersection with LOS). The application requirements are satisfied if $\mathrm{D}_{\mathrm{p}}>0$. The vertical lines represent the $5^{\text {th }}$ and $95^{\text {th }}$ percentiles.

Fig. 4 and 5 represent a snapshot of the packet transmission frequency and power used by each vehicle after 50 seconds of simulation time. INTERN and MINT adapt the vehicles' packet transmission frequency based on their individual application requirements. This results in that vehicles implementing INTERN and MINT utilize very different packet transmission frequency and power (Fig. 4 and Fig. 5). On the other hand, the use of LIMERIC+PULSAR results in that all vehicles always use the same power, but their packet transmission frequency is adapted so that similar values are utilized by neighboring vehicles. Fig. $4 \mathrm{~b}$ shows how reducing the transmission power 
with LIMERIC+PULSAR allows increasing the packet transmission frequency. For $P_{t}=13 \mathrm{dBm}$, most of the vehicles are configured with the maximum packet transmission frequency considered in this study $\left(T_{f}=20 \mathrm{~Hz}\right)$.

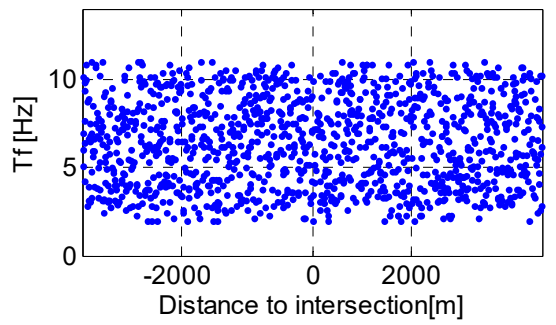

(a) MINT

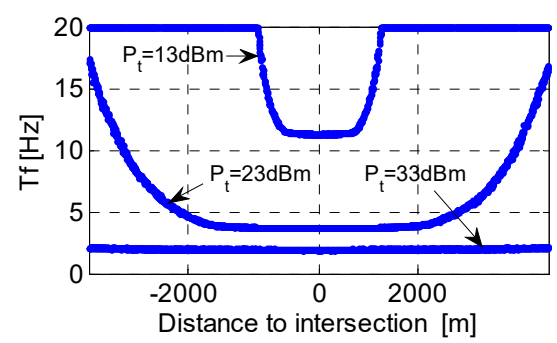

(b) LIMERIC+PULSAR

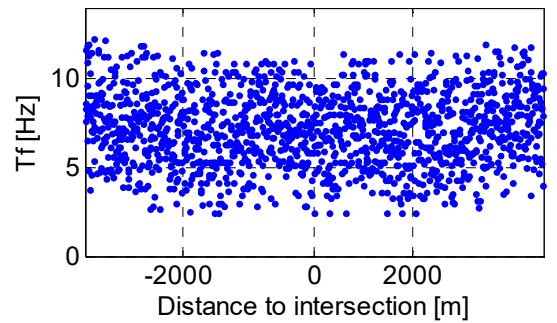

(c) INTERN

Fig. 4. Snapshot of the packet transmission frequency used by each vehicle after 50s of simulation time. Scenario 1 and a traffic density of $50 \mathrm{veh} / \mathrm{km} / \mathrm{lane}$.

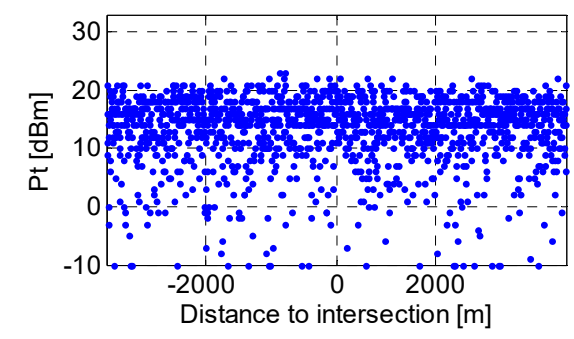

(a) MINT

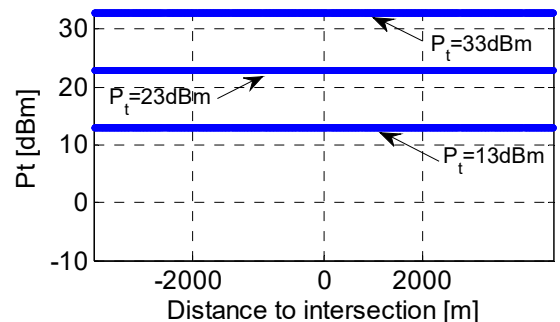

(b) LIMERIC+PULSAR

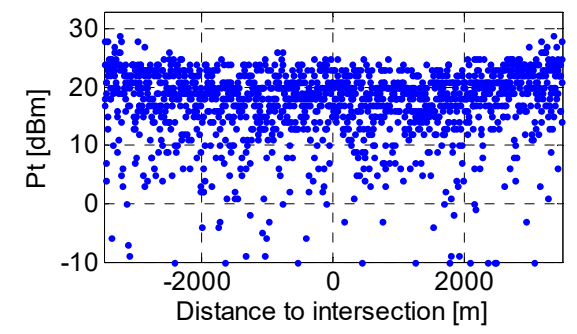

(c) INTERN

Fig. 5. Snapshot of the transmission power used by each vehicle after 50s of simulation time. Scenario 1 and a traffic density of 50 $\mathrm{veh} / \mathrm{km} / \mathrm{lane}$.

The variability shown in Fig. 4 and 5 for the packet transmission frequency and power utilized when implementing INTERN is due to the fact that the application requirements for each vehicle have been randomly selected so that the study is not restricted to a particular application. Another way of observing the performance of the proposal is to look into the stability of the $D_{p}$ metric for two selected vehicles. This is shown in Fig. 6 . The oscillations of the $D_{p}$ metric are due to INTERN's control process and are in the same order or lower than e.g. the variations in packet transmission frequency reported in the literature [14].

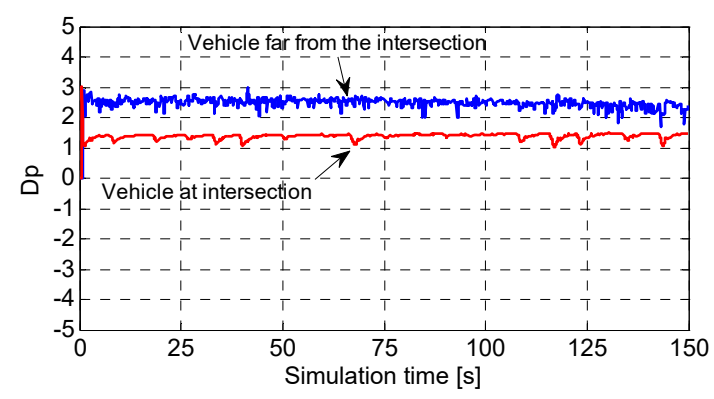

Fig. 6. Stability of the $D_{p}$ metric with INTERN for two selected vehicles. Scenario 1 and a traffic density of $50 \mathrm{veh} / \mathrm{km} / \mathrm{lane}$. 


\section{Scenario 2 - intersection with NLOS}

The second scenario is characterized by the presence of buildings at the intersection corners. These buildings block radio propagation and can severely impact the communications between vehicles approaching the intersection from different directions. This has been shown, for example, to have an important negative effect for applications such as intersection collision warning [8]. However, the signal attenuation produced by buildings also reduces the channel load levels experienced by the majority of vehicles in the scenario. This is the case because vehicles can only detect packets transmitted by other vehicles under NLOS conditions if they are at short distances. In scenario 2, vehicles located at the intersection have LOS conditions with all vehicles in the scenario, and therefore are not affected by the attenuation produced buildings.

Figures 7 and 8 plot the average spatial distribution of the $C B R$ and $D_{p}$ as a function of the distance of the vehicles to the intersection. The results are plotted for different traffic densities in scenario 2, and the vertical lines represent the $5^{\text {th }}$ and $95^{\text {th }}$ percentiles. The comparison of Fig. 2 and Fig. 7 shows that MINT produces similar channel load levels for vehicles located at medium and high distances to the intersection in scenarios 1 and 2 . This is the case because MINT adapts the transmission parameters of each vehicle to the minimum needed to satisfy its requirements. As a result, vehicles at medium and high distances to the intersection do not detect vehicles at perpendicular streets independent of whether there are buildings at the intersection corners or not. However, the channel load experienced by vehicles close to the intersection is significantly different for scenarios 1 and 2 . Fig. $7 \mathrm{a}$ shows that only vehicles located at the intersection experience high CBR levels when implementing MINT in scenario 2. The results in Fig. 8a show that the same conclusions can be reached in scenario 1 and 2 with regards to the impact of MINT on the $D_{p}$ metric: the application requirements are satisfied with a constant $D_{p}$ metric independent of the traffic density.

LIMERIC+PULSAR uses a fixed transmission power and adapts the packet transmission frequency of each vehicle to maintain the $C B R$ below $C B R_{\max }$. In scenario 1, the best results for LIMERIC+PULSAR in terms of application' effectiveness were obtained with the lowest considered transmission power. The results obtained by LIMERIC+PULSAR in scenario 2 are then only shown for $P_{t}=13 \mathrm{dBm}$. The packet transmission frequency obtained with LIMERIC+PULSAR in scenario 2 is similar to the one obtained in scenario 1 . This is due to the fact that vehicles adapt their packet transmission frequency based on the highest $C B R$ experienced at 2 hops. This $C B R$ corresponds to the one experienced at the intersection, and that is less affected by the presence of the obstructing buildings. As a consequence, the presence of buildings in scenario 2 results in a reduction of the channel load levels obtained with LIMERIC+PULSAR (Fig. 7b) compared to scenario 1 except for vehicles located at the intersection. Since vehicles use similar packet transmission frequency values in scenarios 1 and 2, no significant differences are observed with regards to the experienced $D_{p}$ levels between both scenarios.

Compared to MINT, INTERN increases the transmission parameters until the $C B R_{\max }$ level is reached at the center of the intersection. The results in Fig. 7c show that INTERN is capable to maintain the $C B R$ below $C B R_{\max }$ in scenario 2. Fig. 7c also shows that vehicles experiencing low $C B R$ values do not increase their transmission parameters to avoid augmenting the $C B R$ experienced by vehicles close to the intersection. The presence of buildings in scenario 2 results in that INTERN can increase the vehicles' transmission parameters compared to scenario 1 . This explains why higher $D_{p}$ levels are obtained in scenario 2 (Fig. 8c) compared to scenario 1 (Fig. 3).

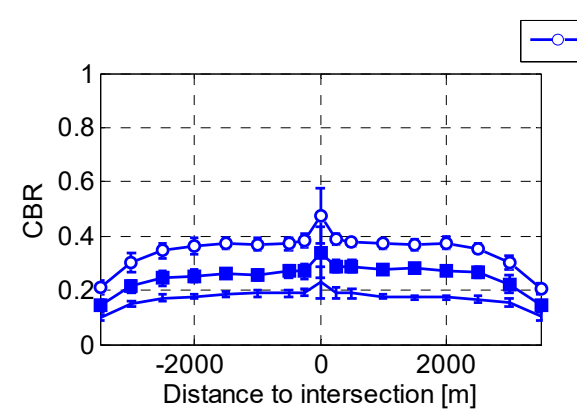

(a) MINT

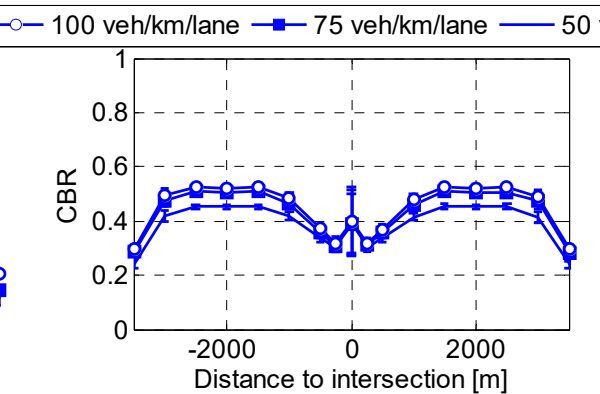

(b) LIMERIC+PULSAR $\left(\mathrm{P}_{\mathrm{t}}=13 \mathrm{dBm}\right)$

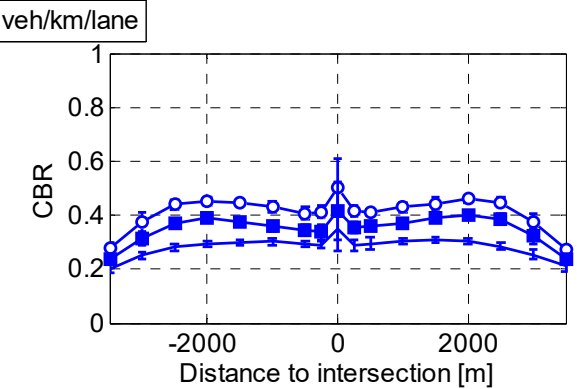

(c) INTERN

Fig. 7. Average spatial distribution of the $C B R$ (Channel Busy Ratio) under different traffic densities in scenario 2 (intersection with NLOS). The vertical lines represent the $5^{\text {th }}$ and $95^{\text {th }}$ percentiles. 


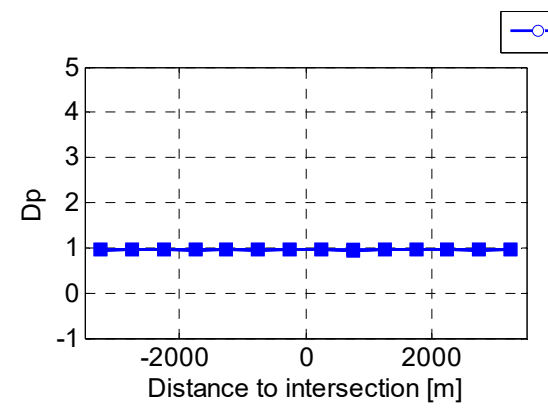

(a) MINT

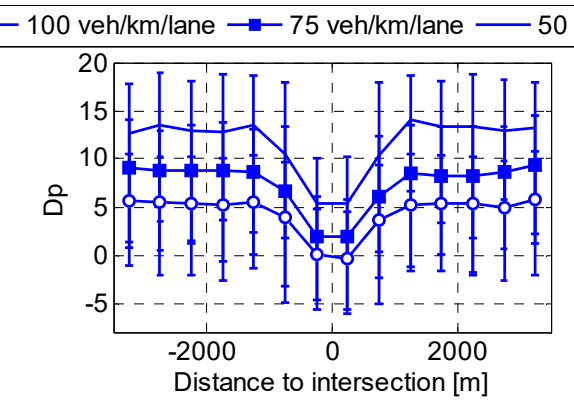

(b) LIMERIC + PULSAR $\left(\mathrm{P}_{\mathrm{t}}=13 \mathrm{dBm}\right)$

$50 \mathrm{veh} / \mathrm{km} / \mathrm{lane}$

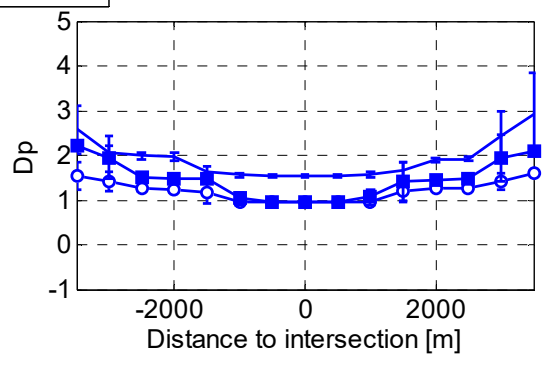

(c) INTERN

Fig. 8. Average spatial distribution of the $\mathrm{D}_{\mathrm{p}}$ metric under different traffic densities in scenario 2 (intersection with NLOS). The application requirements are satisfied if $\mathrm{D}_{\mathrm{p}}>0$. The vertical lines represent the $5^{\text {th }}$ and $95^{\text {th }}$ percentiles.

\section{Scenario 3 - highway}

Scenario 3 was selected to analyze the stability and convergence of the protocols. Fig. 9 and 10 plot the $C B R$ and $D_{p}$ metrics experienced in scenario 3 as a function of the position of the vehicles in the highway (Position $=0$ represents the center of the scenario). The metrics are depicted for a traffic density of $75 \mathrm{veh} / \mathrm{km} / \mathrm{lane}$ and time instants $t=25 \mathrm{~s}, t=50 \mathrm{~s}$ (when approximately the two groups of vehicles reach the center of the scenario) and $t=75 \mathrm{~s}$ (when the two groups of vehicles are aligned one next to each other).

Fig. 9a shows that the $C B R$ increases as the two groups of vehicles approach the center of the scenario when utilizing MINT. This is due to the fact that vehicles implementing MINT do not adapt their parameters as a function of the experienced channel load. As a result, when the two groups are far from each other $(t=25 \mathrm{~s})$, the CBR is much lower than when the two groups of vehicles are aligned $(t=75 \mathrm{~s})$. Since vehicles always use the minimum required transmission settings, the $D_{p}$ metric is constant during the simulation (Fig. 10a). Similar trends have been obtained for $50 \mathrm{veh} / \mathrm{km} / \mathrm{lane}$ despite the different experienced channel load levels. When the traffic density increases to $100 \mathrm{veh} / \mathrm{km} / \mathrm{lane}$, the target $C B R_{\max }$ level is exceeded at the center of the scenario when the two groups of vehicles align at $t=75 \mathrm{~s}$. This result demonstrates that it is not possible either to satisfy the application requirements of all vehicles in scenario 3 without exceeding $C B R_{\max }$.

Vehicles implementing LIMERIC+PULSAR transmit at a constant transmission power level and adapt the packet transmission frequency based on the experienced channel load. Fig. 9b shows that LIMERIC+PULSAR maintain the maximum $C B R$ level below $C B R_{\max }$ as the two groups of vehicles approach each other. With LIMERIC+PULSAR, neighboring vehicles tend to use similar packet transmission frequencies. Since they can have different application requirements, they can experience different $D_{p}$ levels (Fig. 10b) and negative values can be observed at $t=75 \mathrm{~s}$ (i.e. when the two groups of vehicles are aligned).

With INTERN, vehicles adapt their transmission parameters based on their individual application requirements and the channel load experienced. At $t=25 \mathrm{~s}$, the two groups of vehicles are far from each other and the experienced channel load is low (Fig. 9c). In this case, vehicles operate at the maximum $\Delta T_{f}$ and constant $D_{p}$ values are obtained (Fig. 10c). As the two groups of vehicles approach each other, the channel load increases and the $\Delta T_{f}$ parameter decreases, which results in lower but still positive $D_{p}$ metric. Using the information exchange process that is integrated into INTERN, nearby vehicles exhibit very similar $D_{p}$ values, thereby satisfying the objective of fairness at the application level. Similar trends have been obtained for $50 \mathrm{veh} / \mathrm{km} /$ lane: the $C B R$ level was also maintained below $C B R_{\max }$ and the $D_{p}$ metric was positive (higher $D_{p}$ levels were observed due to the lower traffic density). When traffic density increases to $100 \mathrm{veh} / \mathrm{km} / \mathrm{lane}$, all vehicles are configured to use the minimum transmission settings they need to satisfy their requirements at $t=75 \mathrm{~s}$. Despite this configuration, the target $C B R_{\max }$ level is again exceeded when the two groups of vehicles are aligned.

After $t=75 \mathrm{~s}$, the two groups of vehicles start moving away from each other. At $t=100 \mathrm{~s}$, the measured channel load and application's effectiveness are similar than the ones experienced at $t=50 \mathrm{~s}$ for all three protocols under evaluation. 
At $t=125 \mathrm{~s}$, the values obtained are similar to the ones experienced at $t=25 \mathrm{~s}$. These results show that the 3 protocols are able to recover from the maximum load experienced at $t=75 \mathrm{~s}$, and return to the initial values as the two groups of vehicles separate from each other.
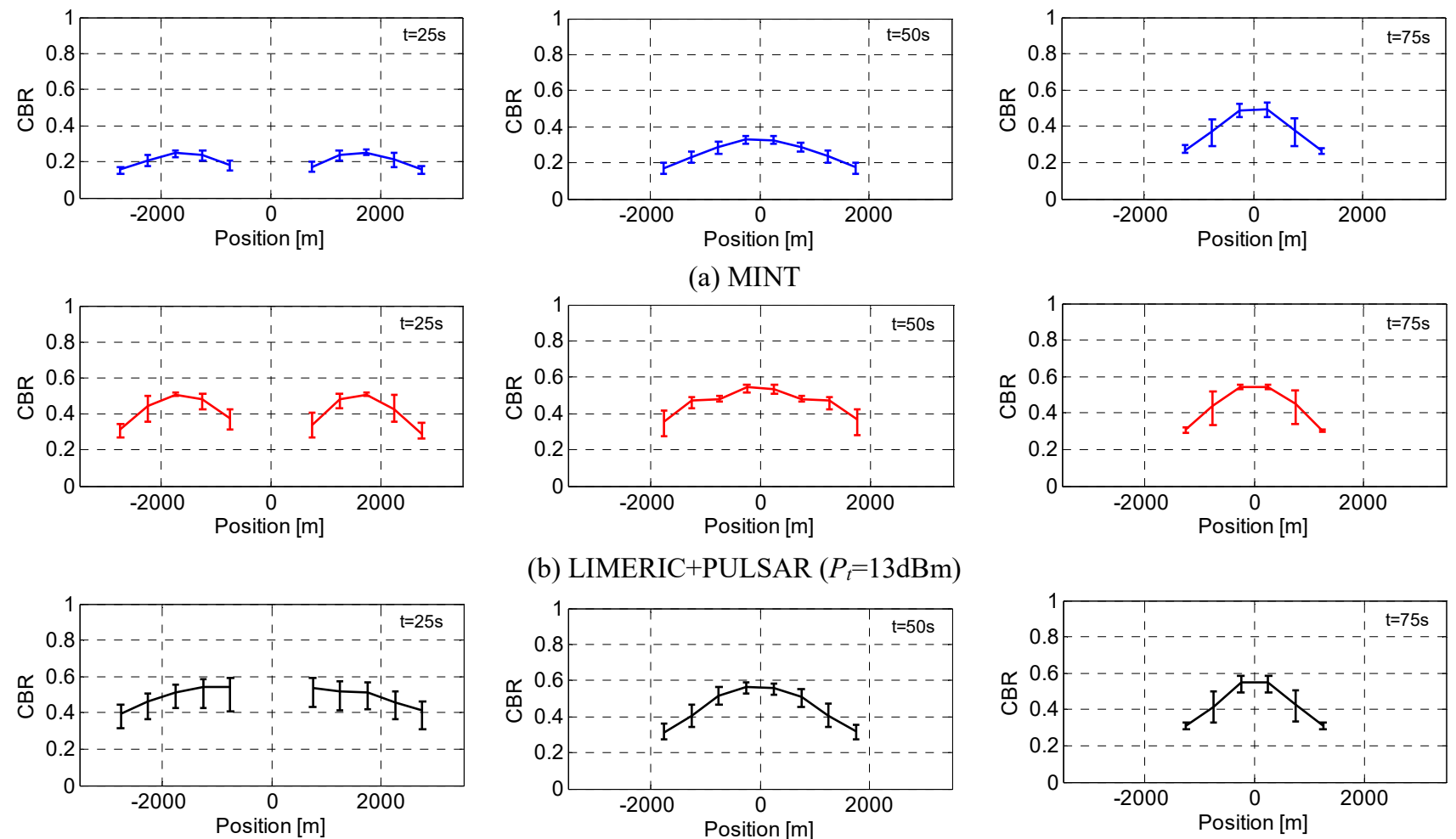

(b) LIMERIC+PULSAR $\left(P_{t}=13 \mathrm{dBm}\right)$
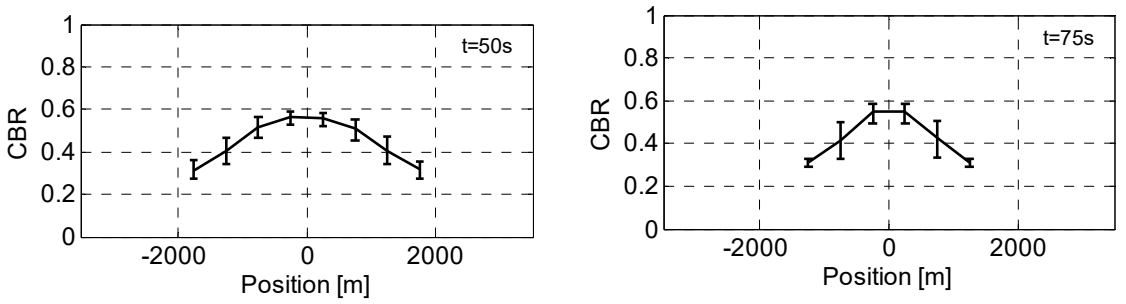

(c) INTERN

Fig. 9. CBR (Channel Busy Ratio) at $t=25 \mathrm{~s}, t=50 \mathrm{~s}$ and $t=75 \mathrm{~s}$. Scenario 3 (highway) and a traffic density of $75 \mathrm{veh} / \mathrm{km} / \mathrm{lane}$. The vertical lines represent the $5^{\text {th }}$ and $95^{\text {th }}$ percentiles. 

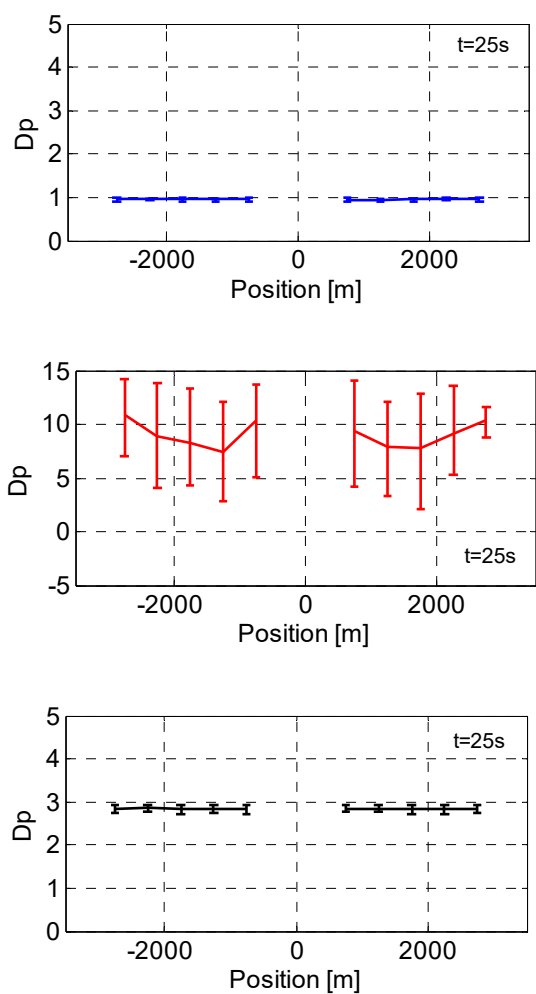

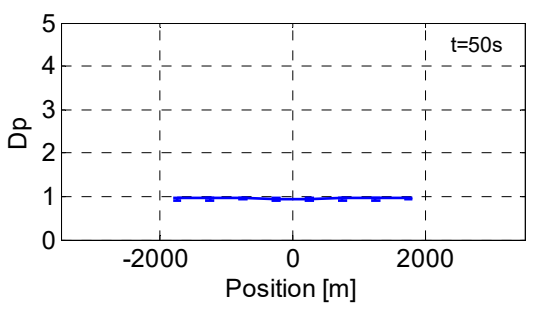

(a) MINT

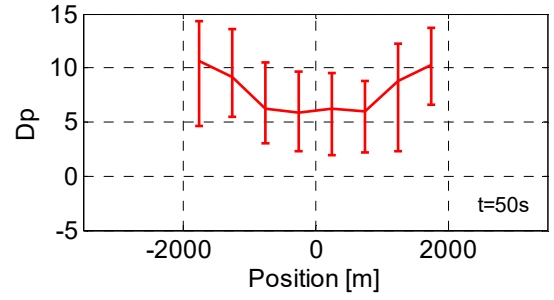

(b) LIMERIC+PULSAR $\left(P_{t}=13 \mathrm{dBm}\right)$

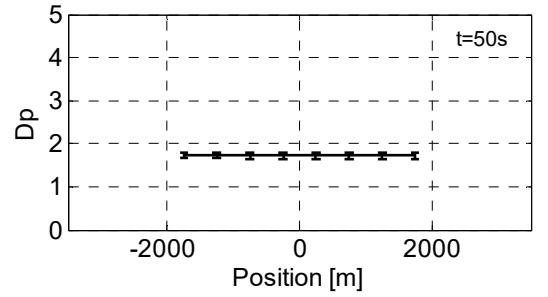

(c) INTERN
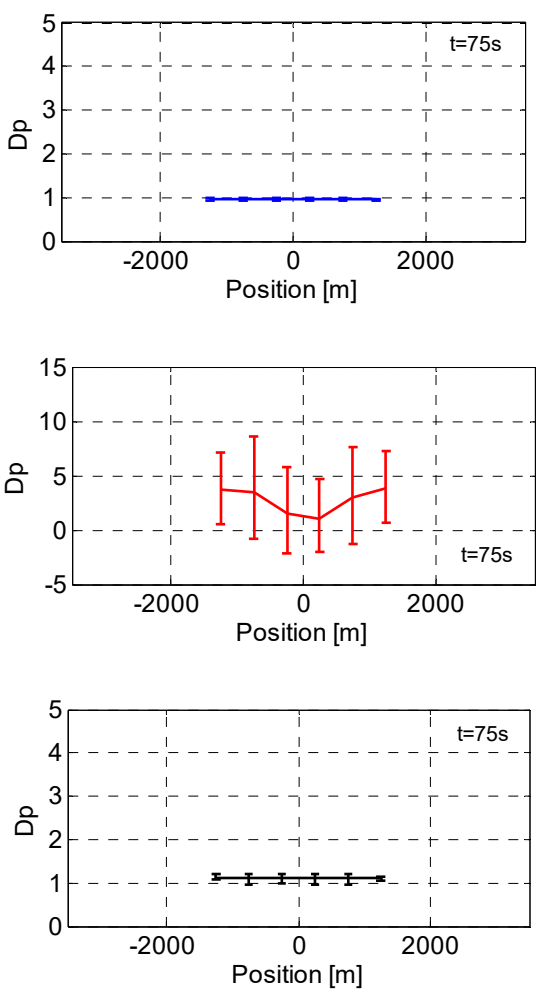

Fig. 10. $\mathrm{D}_{\mathrm{p}}$ metric at $t=25 \mathrm{~s}, t=50 \mathrm{~s}$ and $t=75 \mathrm{~s}$. Scenario 3 (highway) and a traffic density of $75 \mathrm{veh} / \mathrm{km} / \mathrm{lane}$. The vertical lines represent the $5^{\text {th }}$ and $95^{\text {th }}$ percentiles. The application requirements are satisfied if $D_{p}>0$.

Fig. 11 illustrates the evolution of the $D_{p}$ metric with the simulation when vehicles implement the INTERN proposal. The evolution is represented for three selected vehicles in scenario 3 and with different traffic densities. The vehicles were selected at the head, middle and tail of group 1 in scenario 3 (Fig. 1c). After a short initial transition period, INTERN reaches a stable condition, and provides similar and constant $D_{p}$ values to all vehicles. After $t=50$ s, the experienced channel load increases and the $D_{p}$ metric decreases with smooth and similar variations for the three vehicles. The minimum $D_{p}$ values are experienced around $t=75 \mathrm{~s}$ as the two groups of vehicles align; the alignment produces the highest traffic density level in the simulation. After $t=75 \mathrm{~s}$, the traffic density decreases and INTERN is able to recover to the initial $D_{p}$ metrics.

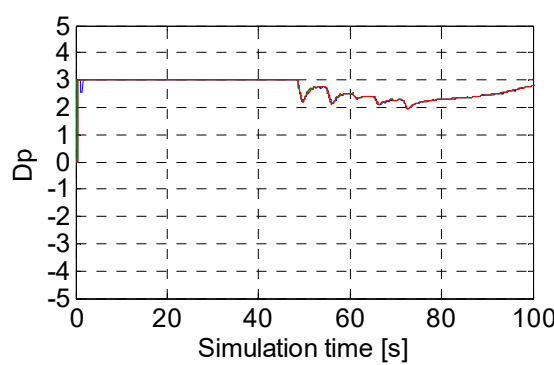

(a) $50 \mathrm{veh} / \mathrm{km} / \mathrm{lane}$

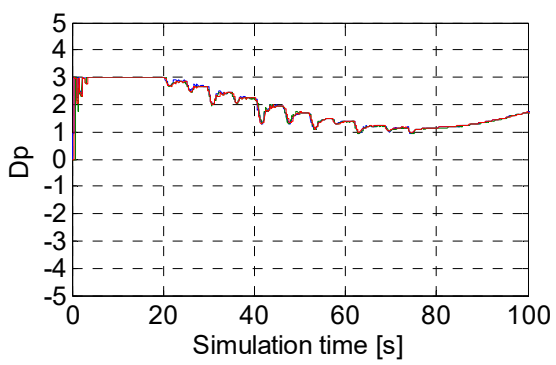

(b) $75 \mathrm{veh} / \mathrm{km} / \mathrm{lane}$

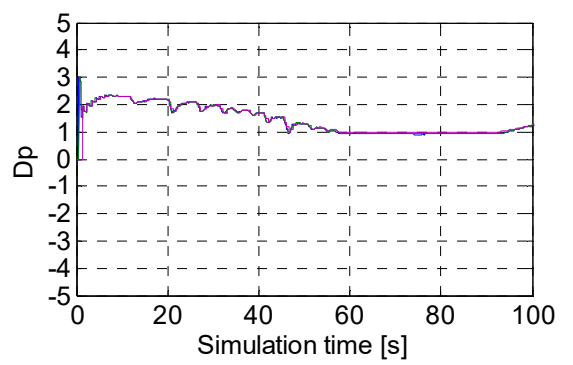

(c) $100 \mathrm{veh} / \mathrm{km} / \mathrm{lane}$

Fig. 11. Stability of the $\mathrm{D}_{\mathrm{p}}$ metric when implementing INTERN. Scenario 3 (highway) and different traffic densities. 


\section{E. Constant and equal application requirements}

Previous sections considered that each vehicle selected randomly its application requirements so that the study was not limited to a particular application. The vehicles selected randomly a warning distance $\left(W_{d}\right)$ between 50 and $200 \mathrm{~m}$, and a packet reception frequency $(R)$ between $1 \mathrm{~Hz}$ and $10 \mathrm{~Hz}$. In this section, we evaluate the performance of the three protocols considering constant and equal application requirements for all vehicles during each simulation run. Different simulation runs have been executed with the warning distance selected within the range of 50 to $150 \mathrm{~m}$, and the packet reception frequency within the range of $1 \mathrm{~Hz}$ to $10 \mathrm{~Hz}$. The objective behind this analysis is to study the influence of the application requirements on the channel load and the applications' effectiveness.

With MINT, each vehicle sets its transmission power and packet transmission frequency to the minimum level needed to satisfy its application requirements. As a result, the experienced channel load is directly influenced by the application requirements, and the application requirements are satisfied with a constant $D_{p}$ metric. Fig. 12 illustrates the average $C B R$ experienced with MINT for varying application requirements and different traffic densities in scenario 1 (intersection with LOS). Fig. 12 shows that augmenting the application requirements $\left(W_{d}\right.$ and $\left.R\right)$ increases the experienced $C B R$ level with MINT since each vehicle adapts its transmission parameters according to its application requirements. The experienced $C B R$ level also increases with the traffic density. Fig. 12 also shows that the impact of the application requirements on the $C B R$ can be important, and high channel load levels can be experienced even at medium traffic densities when applications have high requirements.

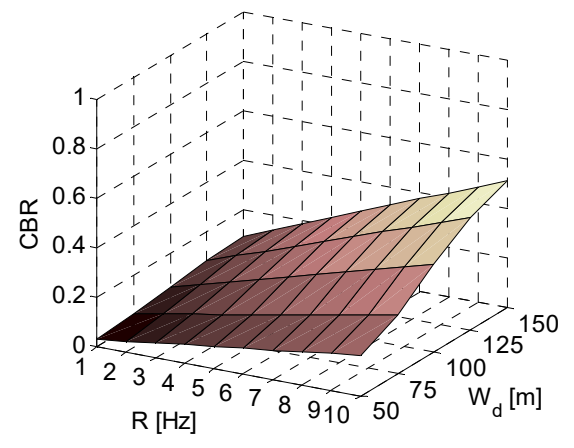

(a) $50 \mathrm{veh} / \mathrm{km} / \mathrm{lane}$

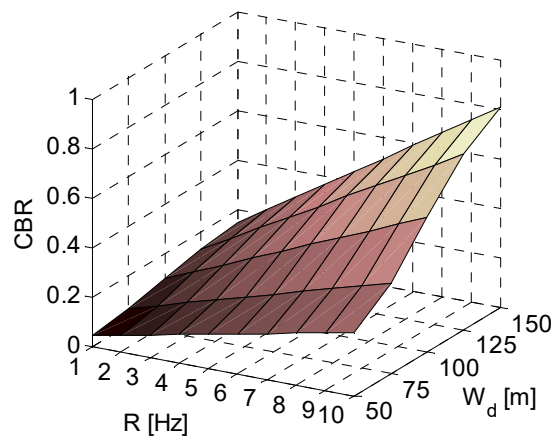

(b) $75 \mathrm{veh} / \mathrm{km} / \mathrm{lane}$

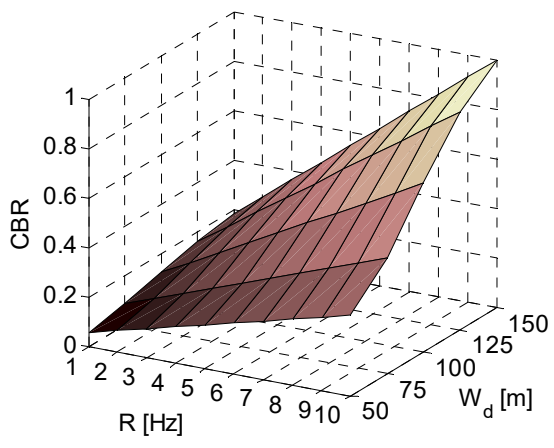

(c) $100 \mathrm{veh} / \mathrm{km} / \mathrm{lane}$

Fig. 12. Average CBR (Channel Busy Ratio) experienced with MINT for different application requirements in scenario 1 (intersection with LOS).

LIMERIC+PULSAR adapts the packet transmission frequency of each vehicle to strictly maintain the $C B R$ below $C B R_{\max }$ irrespective of the traffic density or the application requirements. Fig. 13 depicts the average $D_{p}$ metric obtained with LIMERIC+PULSAR for different application requirements and traffic densities in scenario 1 with $P_{t}=13 \mathrm{dBm}$. Fig. 13 shows that an increment of the required packet reception frequency decreases the average $D_{p}$. This is due to the fact that the $D_{p}$ metric represents the difference between the packet reception frequency required by the application and the number of packets that are actually received per second at the applications' warning distance. With LIMERIC+PULSAR, the number of packets correctly received per second at the warning distance does not change with $R$. As a result, if $R$ is increased, the average $D_{p}$ metric decreases. 


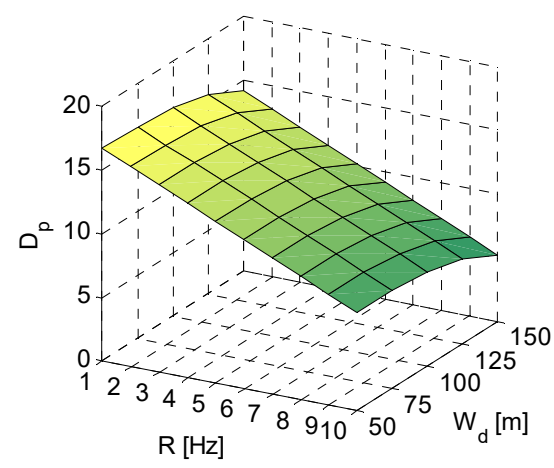

(a) $50 \mathrm{veh} / \mathrm{km} / \mathrm{lane}$

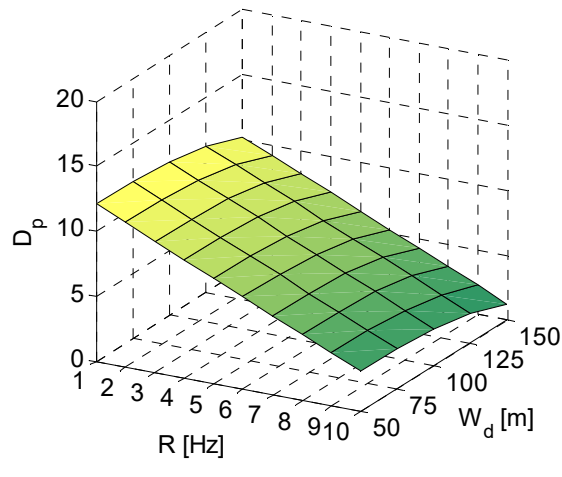

(b) $75 \mathrm{veh} / \mathrm{km} / \mathrm{lane}$

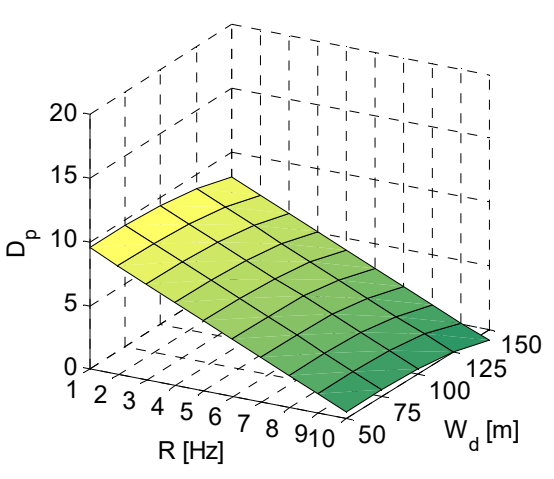

(c) $100 \mathrm{veh} / \mathrm{km} / \mathrm{lane}$

Fig. 13. Average $D_{\mathrm{p}}$ metric experienced with LIMERIC + PULSAR $\left(P_{t}=13 \mathrm{dBm}\right)$ for different application requirements in scenario 1 (intersection with LOS).

Fig. 14 and Fig. 15 represent the average $C B R$ and $D_{p}$ obtained with INTERN. When the requirements and the traffic density are low, INTERN increases the transmission settings to all vehicles, which improves their $D_{p}$ metric accordingly. Fig. 15 shows that the maximum $D_{p}$ value ( $D_{p}=3$ in this study) is obtained with the lowest application requirements. On the other hand, INTERN sets each vehicle's parameters to the minimum needed to satisfy their requirements and minimize the channel load when the traffic density and application requirements increase to their maximum simulated values. The experienced $C B R$ can surpass $C B R_{\max }$ when the requirements are high, although in that case the vehicles are configured with the minimum parameters needed to satisfy their application's requirements. This result demonstrates that in this situation it is not possible to satisfy the application requirements of all vehicles without exceeding the target $C B R_{\max }$ value.

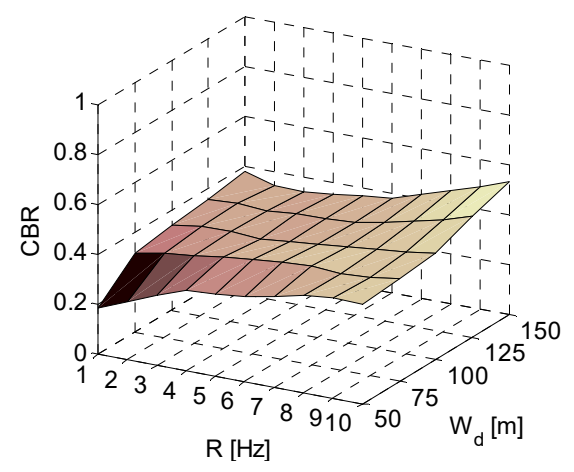

(a) $50 \mathrm{veh} / \mathrm{km} / \mathrm{lane}$

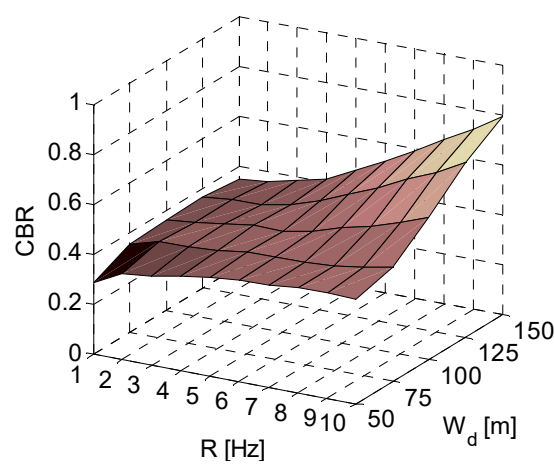

(b) $75 \mathrm{veh} / \mathrm{km} / \mathrm{lane}$

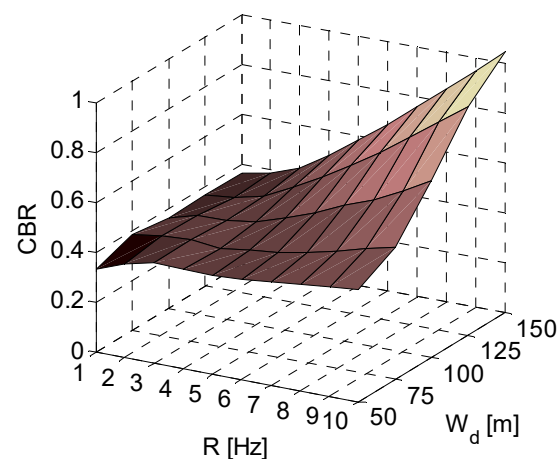

(c) $100 \mathrm{veh} / \mathrm{km} / \mathrm{lane}$

Fig. 14. Average CBR (Channel Busy Ratio) experienced with INTERN for different application requirements in scenario 1 (intersection with LOS). 


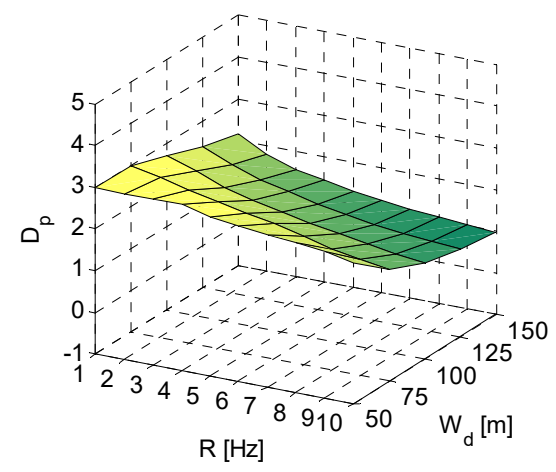

(a) $50 \mathrm{veh} / \mathrm{km} / \mathrm{lane}$

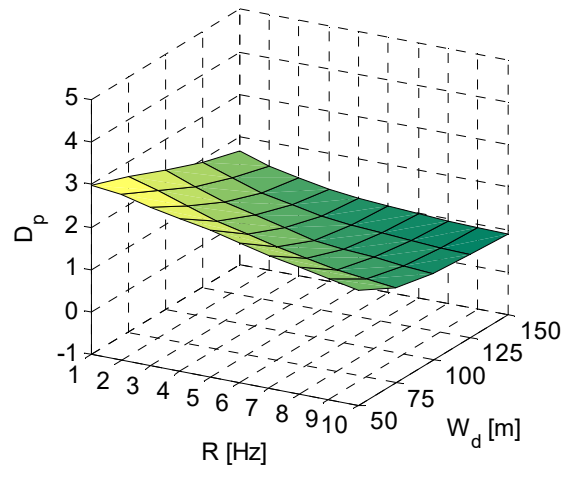

(b) $75 \mathrm{veh} / \mathrm{km} / \mathrm{lane}$

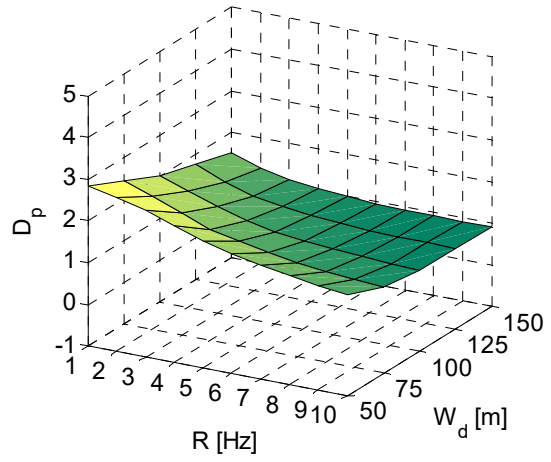

(c) $100 \mathrm{veh} / \mathrm{km} / \mathrm{lane}$

Fig. 15. Average $D_{p}$ metric experienced with INTERN for different application requirements in scenario 1 (intersection with LOS).

\section{DISCUSSION}

The results reported in this paper have shown the relevant influence of the transmission parameters on the channel load generated and the applications' effectiveness. Congestion control protocols that solely adapt the transmission parameters to control the channel load can hence have an important influence on the applications' effectiveness. Similarly, awareness control protocols that adapt the transmission parameters to control the capacity of each vehicle to communicate with nearby vehicles can also influence the experienced channel load. This study therefore suggests that congestion and awareness control protocols can be considered together. Existing protocols such as LIMERIC are being evolved in this direction to differentiate the requirements from different vehicles. For example, EMBARC [29] adapts each vehicle's packet transmission frequency using LIMERIC, but allows specific vehicles (e.g. highly dynamic vehicles) to transmit additional packets to reduce the tracking error of surrounding vehicles. With EMBARC, the target channel load can be maintained by reducing the packet transmission frequency to vehicles that do not have stringent needs. Specific vehicles can then be prioritized. LIMERIC was also extended in [30] to converge to weighted fair packet transmission frequencies. With this extension, two (or more) vehicles could converge to a desired ratio of packet transmission frequencies, if such ratio is known. This approach enables differentiating vehicles with different needs while controlling the channel load. For example, emergency vehicles could be provided with double packet transmission frequency than the rest of vehicles.

Another way of looking into the protocol performance is presented in Fig. 16 and Fig. 17. These figures identify the feasible regions for scenario 1 (intersection with LOS) considering INTERN (the same feasible regions are obtained with MINT) and LIMERIC+PULSAR, respectively. The figures consider that all vehicles have equal and constant application requirements. The figures depict a score (between 0 and 1) for each combination of warning distance and packet reception frequency required by the application. This score is equal to the proportion of vehicles experiencing a channel load below $C B R_{\max }=0.6$ and an application's effectiveness $D_{p}>0$. As a result, a score equal to 1 is obtained when the requirements of all vehicles could be satisfied without overloading the channel (i.e. without exceeding $C B R_{\max }$ ). Feasible regions are hence defined as the regions (i.e. the combinations of $W_{d}$ and $R$ ) with a score equal to 1 . Fig. 16 and Fig. 17 show the feasible regions that can be obtained with INTERN and LIMERIC+PULSAR for various traffic densities. Despite the significant improvements provided by both protocols, Fig. 16 and Fig. 17 also show the difficulties to ubiquitously satisfy the application requirements when operating under medium and high traffic densities. A higher number of lanes or packet payload could in fact further increase the limitations observed in Fig. 16 and Fig. 17. 


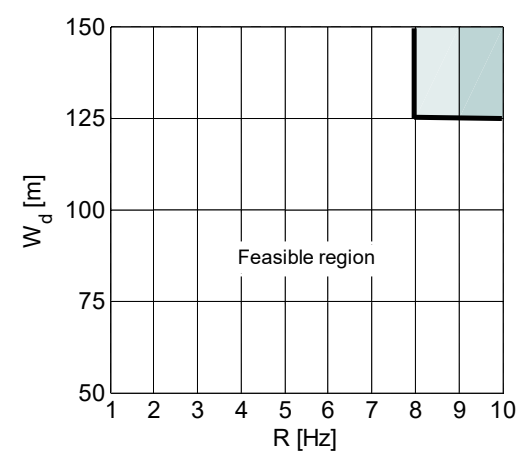

(a) $50 \mathrm{veh} / \mathrm{km} / \mathrm{lane}$

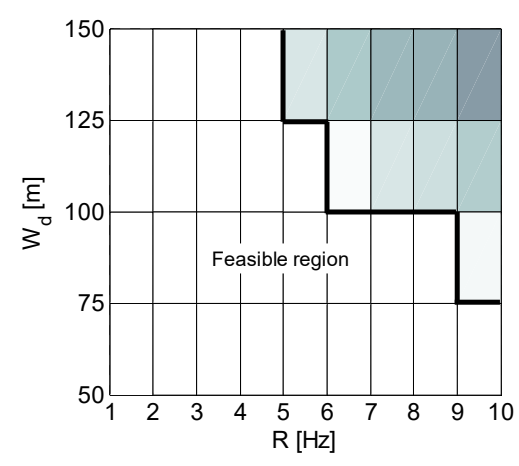

(b) $75 \mathrm{veh} / \mathrm{km} / \mathrm{lane}$

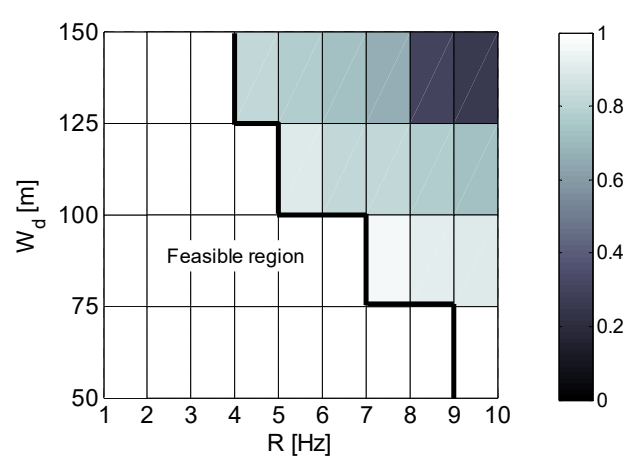

(c) $100 \mathrm{veh} / \mathrm{km} / \mathrm{lane}$

Fig. 16. Feasible regions for different combinations of application requirements considering INTERN. The results are shown for different traffic densities under scenario 1 (intersection with LOS). Each region is colored based on the proportion of vehicles experiencing a channel load below $C B R_{\max }=0.6$ and an application's effectiveness $D_{p}>0$.

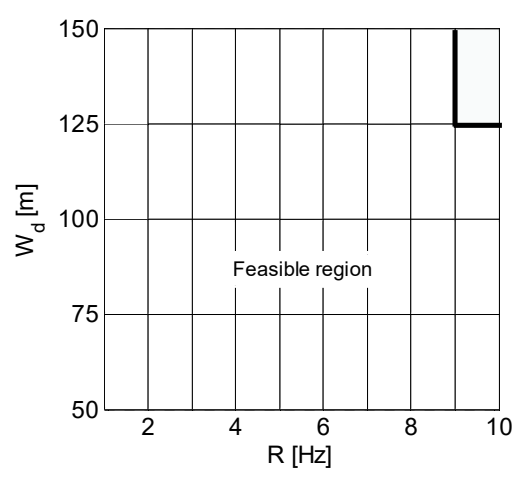

(a) $50 \mathrm{veh} / \mathrm{km} / \mathrm{lane}$

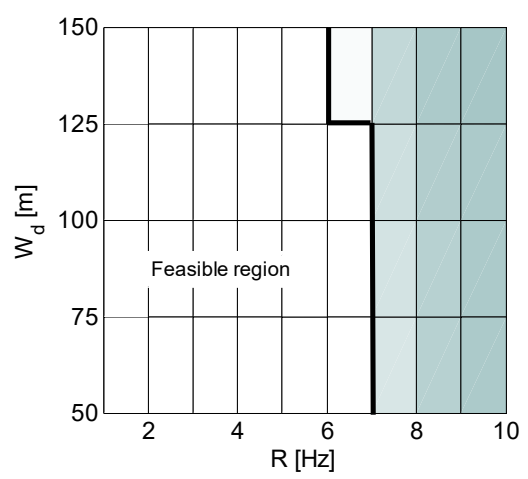

(b) $75 \mathrm{veh} / \mathrm{km} / \mathrm{lane}$

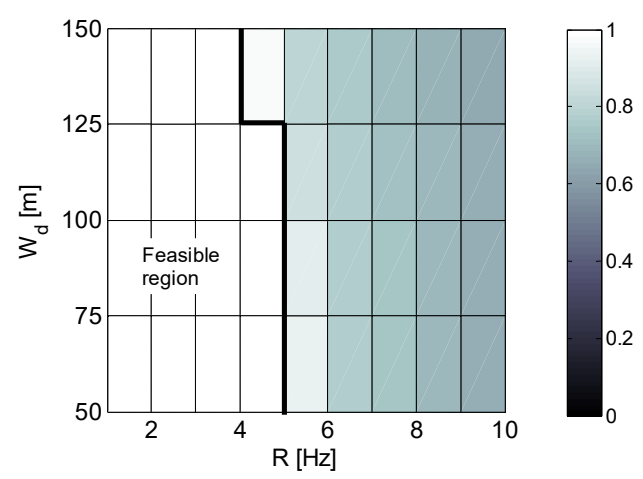

(c) $100 \mathrm{veh} / \mathrm{km} / \mathrm{lane}$

Fig. 17. Feasible regions for different combinations of application requirements considering LIMERIC+PULSAR. The results are shown for different traffic densities under scenario 1 (intersection with LOS). Each region is colored based on the proportion of vehicles experiencing a channel load below $C B R_{\max }=0.6$ and an application's effectiveness $D_{p}>0$.

An additional communications challenge will also emerge with the introduction of future automated and connected vehicles. First, the relation between relative speed and inter-vehicle distance will notably change when vehicles are automatically driven, so higher traffic densities at higher speeds might be possible. Automated vehicles could also require the exchange of richer information between vehicles, for example, sensors (including video) information. Cooperative perception technology is currently being investigated to increase situational awareness for cooperative automated driving. Recent studies suggest that cooperative perception could require the transmission of processed images and laser scans 20 times per second [31], which would notably increase the channel load and the communication requirements of future automated and connected vehicles.

\section{CONCLUSIONS}

This paper has proposed and evaluated INTERN, an integrated congestion and awareness control protocol that dynamically adapts the transmission parameters taking into account the vehicle's application requirements and the experienced channel load. The obtained results demonstrate that INTERN is able to maintain the channel load under control while ensuring that the application requirements of each vehicle are satisfied. The conducted investigation has also shown that INTERN can maintain stable levels of the channel load and the applications' effectiveness. Moreover, INTERN is able to dynamically adapt its operation to variations in traffic density and in terms of application requirements. The study has also highlighted the challenges ahead with emerging automated vehicles. Further 
This is an author-created postprint version. The final publication is available at: https://www.sciencedirect.com/science/article/pii/S1570870515002279

investigations are hence needed to efficiently and reliably support the communication requirements of future vehicular applications, which could be especially demanding with the introduction of automated connected vehicles.

\section{ACKNOWLEDGEMENT}

The authors would like to thank Dr. John Kenney for his valuable comments and suggestions.

\section{REFERENCES}

[1] IEEE 802.11p-2010: Wireless LAN Medium Access Control (MAC) and Physical Layer (PHY) Specifications Amendment 6: Wireless Access in Vehicular Environments, IEEE Standards Association, 2010.

[2] N. An, M. Maile, D. Jiang, J. Mittag, H. Hartenstein, "Balancing the Requirements for a Zero False Positive/Negative Forward Collision Warning", Proc. Annual Conference on Wireless On-Demand Network Systems and Services (WONS), Banff, Alberta, Canada, pp. 191-195, March 2013.

[3] T. Tielert, D. Jiang, H. Hartenstein, L. Delgrossi, "Joint power/rate congestion control optimizing packet reception in vehicle safety communications", Proc. ACM international workshop on Vehicular inter-networking, systems, and applications (VANET), Taipei, Taiwan, pp. 51-60, 25 June 2013.

[4] M. Sepulcre, J. Gozalvez, "On the importance of application requirements in cooperative vehicular communications", Proc. Int. Conf. on Wireless On-Demand Network Systems and Services (WONS), Bardonecchia, Italy, pp. 124-131, 6-28 Jan. 2011.

[5] M. Sepulcre, J. Gozalvez, J. Härri, H. Hartenstein, "Contextual Communications Congestion Control for Cooperative Vehicular Networks”, IEEE Transactions on Wireless Communications, vol. 10 (2), pp. 385-389, Feb. 2011.

[6] M. Sepulcre, J. Mittag, P. Santi, H. Hartenstein, J. Gozalvez, "Congestion and Awareness Control in Cooperative Vehicular Systems", Proceedings of the IEEE, vol. 99 (7), pp. 1260-1279, July 2011.

[7] ETSI TC ITS, "Intelligent Transport Systems (ITS); Cross Layer DCC Management Entity for operation in the ITS G5A and ITS G5B medium; Report on Cross layer DCC algorithms and performance evaluation”, ETSI TR 101 612 V1.1.1, Sept. 2014.

[8] J. Gozalvez, M. Sepulcre, "Opportunistic technique for efficient wireless vehicular communications", IEEE Vehicular Technology Magazine, vol. 2 (4), pp. 33-39, Dec. 2007.

[9] B. Kloiber, J. Haerri, T. Strang, S. Sand, C. Rico-Garcia, "Random Transmit Power Control for DSRC and its Application to Cooperative Safety", IEEE Transactions on Dependable and Secure Computing, Early Access.

[10] M. Ruffini, H.-J. Reumerman, "Power-rate adaptation in high-mobility distributed ad-hoc wireless networks", Proc. IEEE Vehicular Technology Conference (VTC-Spring), Stockholm, Sweden, pp. 2299-2303, May 2005.

[11] S. Rezaei, R. Sengupta, H. Krishnan, X. Guan, R. Bhatia, "Tracking the position of neighboring vehicles using wireless communications", Transportation Research Part C: Emerging Technologies, vol. 18 (3), pp. 335-350, June 2010.

[12] F.J. Ros, P.M. Ruiz, I. Stojmenovic, "Reliable and Efficient Broadcasting in Vehicular Ad Hoc Networks", Proc. IEEE 69th Vehicular Technology Conference (VTC-Spring), Barcelona, Spain, pp. 1-5, 26-29 April 2009.

[13] J. Mittag, F. Thomas, J. Harri, H. Hartenstein, "A Comparison of Single- and Multi-Hop Beaconing in VANETs", Proc. ACM International Workshop on VehiculAr Inter-NETworking (VANET), Beijing, China, pp. 69-78, 25 Sept. 2009.

[14] J. B. Kenney, G. Bansal, C. E. Rohrs, "LIMERIC: a linear message rate control algorithm for vehicular DSRC systems", Proc. ACM international workshop on Vehicular inter-networking, systems, and applications (VANET), Las Vegas. USA, pp. 21-30, 23 Sept. 2011.

[15] T. Tielert, D. Jiang, Q. Chen, L. Delgrossi, H. Hartenstein, "Design Methodology and Evaluation of Rate Adaptation Based Congestion Control for Vehicle Safety Communications", Proc. IEEE Vehicular Networking Conference (VNC), Amsterdam, Netherlands, pp. 116-123, 14-16 Nov. 2011.

[16] ETSI TC ITS, "Intelligent Transport Systems (ITS); Decentralized Congestion Control Mechanisms for Intelligent Transport Systems operating in the $5 \mathrm{GHz}$ range; Access layer part", ETSI TS 102687 V1.1.1, July 2011.

[17] ETSI TC ITS, "Intelligent Transport Systems (ITS); Vehicular Communications; GeoNetworking; Part 4: Geographical addressing and forwarding for point-to-point and point-to-m ultipoint communications; Sub-part 2: Media-dependent functionalities for ITS-G5”, ETSI TS 102 636-4-2 V1.1.1, Oct. 2013.

[18] M. Torrent-Moreno, P. Santi, H. Hartenstein, "Distributed fair transmit power adjustment for vehicular ad hoc networks", Proc. IEEE Communications Society on Sensor and Ad Hoc Communications and Networks (SECON), Reston, USA, pp. 479-488, 25-28 Sept. 2006.

M. Sepulcre, J. Gozalvez, Onur Altintas and Haris Kremo, "Integration of congestion and awareness control in vehicular networks", Ad Hoc Networks, vol. 37, part 1, pp. 29-43, February 2016. https://doi.org/10.1016/j.adhoc.2015.09.010 
This is an author-created postprint version. The final publication is available at: https:/www.sciencedirect.com/science/article/pii/S1570870515002279

[19] C. Sommer, O.K. Tonguz, F. Dressler, "Traffic information systems: efficient message dissemination via adaptive beaconing”, IEEE Communications Magazine, vol. 49, no. 5, pp. 173-179, May 2011.

[20] C. Sommer, S. Joerer, M. Segata, O.K. Tonguz, R.L. Cigno, F. Dressler, "How Shadowing Hurts Vehicular Communications and How Dynamic Beaconing Can Help", IEEE Transactions on Mobile Computing, vol. 14, no. 7, pp. 1411-1421, July 2015.

[21] X. Shen, X. Cheng, R. Zhang, B. Jiao, Y. Yang, "Distributed Congestion Control Approaches for the IEEE 802.11p Vehicular Networks", IEEE Intelligent Transportation Systems Magazine, vol. 5, no. 4, pp. 50-61, Winter 2013.

[22] Y. Zang, L. Stibor, X. Cheng, H.-J. Reumerman, A. Paruzel, A. Barroso, "Congestion Control in Wireless Networks for Vehicular Safety Applications", Proc. European Wireless Conference, Paris, France, p. 7, 1-4 April 2007.

[23] M.S. Bouassida, M. Shawky, “On the congestion control within VANET”, Proc. IFIP Wireless Days, pp. 1-5, United Arab Emirates, 24-27 Nov. 2008.

[24] M. Sepulcre, J. Gozalvez, J. Härri, H. Hartenstein, “Application-Based Congestion Control Policy for the Communication Channel in VANETs”, IEEE Communications Letters, vol. 14 (10), pp. 951-953, Oct. 2010.

[25] M. Sepulcre, J. Gozalvez, O. Altintas, H. Kremo, “Adaptive Beaconing for Congestion and Awareness Control in Vehicular Networks", Proc. IEEE Vehicular Networking Conference (VNC), pp. 81-88, Paderborn, Germany, 3-5 Dec. 2014.

[26] M. Killat, H. Hartenstein, "An empirical model for probability of packet reception in vehicular ad hoc networks", EURASIP Journal on Wireless Communications and Networking, pp. 1-12, Jan. 2009.

[27] T. Mangel, O. Klemp and H. Hartenstein, "5.9 GHz inter-vehicle communication at intersections: a validated non-line-ofsight path-loss and fading model”, EURASIP Journal on Wireless Communications and Networking 2011, $2011: 182$.

[28] M. Boban, P. M. d'Orey, "Measurement-based evaluation of cooperative awareness for V2V and V2I communication", Proc. IEEE Vehicular Networking Conference (VNC), Paderborn, Germany, pp. 1-8, 3-5 Dec. 2014.

[29] G. Bansal, H. Lu, J. Kenney and C. Poellabauer, "EMBARC: error model based adaptive rate control for vehicle-to-vehicle communications", Proc. ACM international workshop on Vehicular inter-networking, systems, and applications (VANET), Taipei, Taiwan, pp. 41-50, 25 June 2013.

[30] G. Bansal and J. Kenney, “Achieving Weighted-Fairness in Message Rate-Based Congestion Control for DSRC Systems”, Proc. IEEE International Symposium on Wireless Vehicular Communications (WiVeC), Dresden, Germany, pp. 1-5, 2-3 June 2013.

[31] S.-W. Kim, B. Qin, Z.J. Chong, X. Shen, W. Liu, M.H. Ang, E. Frazzoli, D. Rus, "Multivehicle Cooperative Driving Using Cooperative Perception: Design and Experimental Validation", IEEE Transactions on Intelligent Transportation Systems, vol. 16, no. 2, pp. 663-680, April 2015..

Miguel Sepulcre received a Telecommunications Engineering degree in 2004 and a Ph.D. in Communications Technologies in 2010, both from the University Miguel Hernández of Elche (UMH), Spain. He was awarded by the COIT (Spanish official association of Telecommunication Engineers) with the ONO prize to the best national Ph.D. He has been visiting researcher at ESA in Noordwijk (The Netherlands) in 2004, at KIT in Karlsruhe (Germany) in 2009, and at Toyota ITC in Tokyo (Japan) in 2015. He serves as Associate Editor for IEEE Vehicular Technology Magazine. This work was conducted during his stay at Toyota ITC, but he is now a post-doc researcher at UWICORE (UMH) working in wireless vehicular networks.

Javier Gozalvez received an electronics engineering degree from the Engineering School ENSEIRB (Bordeaux, France), and a PhD in mobile communications from the University of Strathclyde, Glasgow, U.K. Since October 2002, he is with the Miguel Hernández University of Elche, Spain, where he is currently an Associate Professor and Director of the UWICORE laboratory. At UWICORE, he leads research activities in the areas of wireless industrial networks, multi-hop cellular networks, vehicular networks, resource management and heterogeneous networks. He has published over 110 papers in international conferences and journals. He is an elected member to the Board of Governors (2011-2017) and Executive Vice President of the IEEE Vehicular Technology Society. He is an IEEE Distinguished Lecturer for the IEEE Vehicular Technology Society. He currently serves as Mobile Radio Senior Editor of IEEE Vehicular Technology Magazine and as part of the Editorial Board of Elsevier's Computer Network Journal. He is the General Co-Chair for the IEEE VTC-Spring 2015 conference in Glasgow (UK), and was General Co-Chair of the ACM VANET 2013, ACM VANET 2012 and 3rd ISWCS 2006. He also was TPC Co-Chair for 2011 IEEE VTC-Fall and 2009 IEEE VTC-Spring. He is also the founder and General Co-Chair of the IEEE International Symposium on Wireless Vehicular communications (WiVeC) in its 2007, 2008, and 2010 editions.

M. Sepulcre, J. Gozalvez, Onur Altintas and Haris Kremo, "Integration of congestion and awareness control in vehicular networks", Ad Hoc Networks, vol. 37, part 1, pp. 29-43, February 2016. https://doi.org/10.1016/j.adhoc.2015.09.010 
Onur Altintas is the Fellow at the R\&D Group of Toyota InfoTechnology Center, Co. Ltd, in Tokyo. From 1999 to 2001 he was with Toyota Motor Corporation and was also a visiting researcher at Telcordia Technologies. From 2001 to 2004 he was with Toyota InfoTechnology Center USA. He received his B.S. and M.S. degrees from Orta Dogu Teknik Universitesi, Ankara, Turkey, and his Ph.D. degree from the University of Tokyo, Japan; all in electrical engineering. He is the co-founder and general co-chair of the IEEE Vehicular Networking Conference (IEEE VNC) since 2009. He serves as an associate editor for IEEE ITS Magazine and in the editorial board of Connected Vehicles Series of IEEE Transactions on Vehicular Technology. He is an IEEE VTS Distinguished Lecturer.

Haris Kremo received the Dipl.Ing. degree from the School of Electrical Engineering, University of Sarajevo, Bosnia and Herzegovina in 2000. He received M.S. and Ph.D. degrees from Rutgers, The State University of New Jersey in 2005 and 2010, respectively. From 2000 to 2002 he was a Research Engineer with ENERGOINVEST dd, Sarajevo. From 2003 to 2010 he was a Graduate Assistant with the Wireless Information Networks Laboratory (WINLAB), where he was one of the engineers responsible for design and maintenance of the ORBIT wireless testbed. In 2010 he was with the EDGE Lab, Princeton University as a Postdoctoral Research Associate. Currently he is a Researcher with Toyota InfoTechnology Center, Tokyo. His research interests include dynamic spectrum access and automated driving systems. 